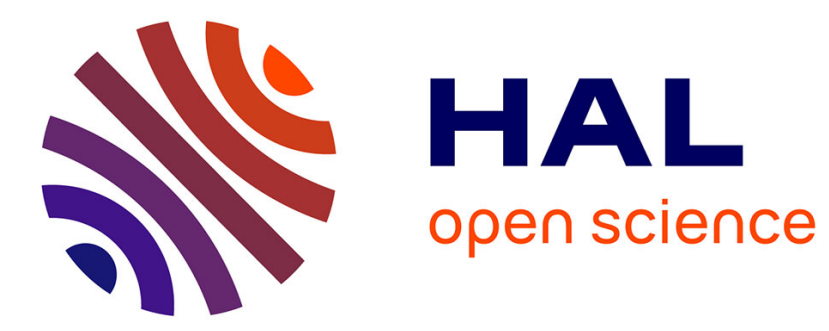

\title{
Dissipative formulation of initial boundary value problems for Friedrichs' systems
}

Clément Mifsud, Bruno Després, Nicolas Seguin

\section{To cite this version:}

Clément Mifsud, Bruno Després, Nicolas Seguin. Dissipative formulation of initial boundary value problems for Friedrichs' systems. Communications in Partial Differential Equations, 2016, 41 (1), 10.1080/03605302.2015.1103750 . hal-01074542

\section{HAL Id: hal-01074542 \\ https://hal.science/hal-01074542}

Submitted on 14 Oct 2014

HAL is a multi-disciplinary open access archive for the deposit and dissemination of scientific research documents, whether they are published or not. The documents may come from teaching and research institutions in France or abroad, or from public or private research centers.
L'archive ouverte pluridisciplinaire $\mathbf{H A L}$, est destinée au dépôt et à la diffusion de documents scientifiques de niveau recherche, publiés ou non, émanant des établissements d'enseignement et de recherche français ou étrangers, des laboratoires publics ou privés. 


\title{
Dissipative formulation of initial boundary value problems for Friedrichs' systems
}

\author{
Clément Mifsud, Bruno Després, Nicolas Seguin
}

October 14, 2014

\begin{abstract}
In this article we present a dissipative definition of a solution for initial boundary value problems for Friedrichs' systems posed in the space $L_{t, x}^{2}$. We study the information contained in this definition and prove an existence and uniqueness theorem in the non-characteristic case and with constant coefficients. Finally, we compare our choice of boundary condition to previous works, especially on the wave equation.
\end{abstract}

\section{Contents}

\begin{tabular}{lll}
\hline 1 & Introduction & 1
\end{tabular}

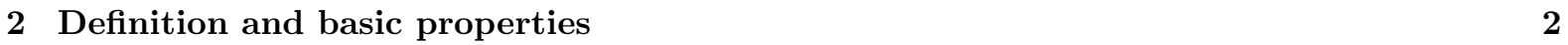

2.1 Motivations and definitions . . . . . . . . . . . . . . . . . . . . 2

2.2 What is the information contained in our formulation? . . . . . . . . . . . . . . . . . 5

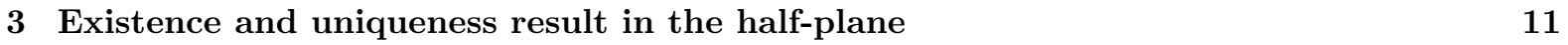

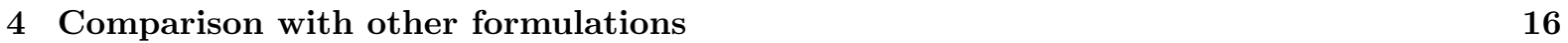

4.1 Friedrichs' approach . . . . . . . . . . . . . . . . . . . . . . . 17

4.2 Dubois-Le Floch's formulation.$\ldots \ldots \ldots \ldots \ldots \ldots \ldots$

4.3 Maximal dissipative and strictly dissipative theories . . . . . . . . . . . . . . . . . . 17

4.4 A simple example: the wave equation in 1D . . . . . . . . . . . . . . . . . . . . . 18

\section{Introduction}

The aim of this paper is to present a notion of solution for initial boundary value problems in the case of linear hyperbolic systems. This problem has been studied by many different authors [9, 10, 15, 14, 2, 18, 17] and there are still a lot of open questions for this kind of problem (in particular in the case of variable multiplicities : see [16]). In addition, many physical systems can recast in a Friedrichs' framework : linear elasticity theory [19, isotropic Maxwell equations [8], linearized Euler equations, MHD [16.

Having in mind dissipative problems such as viscoplastic problems for which boundary conditions play a important mechanical role and their associated limit plastic problems, which can develop singularities and discontinuities, our initial goal was to give a new definition of solution that requires minimal regularity and in particular, the solution does not need a trace on the boundary. In [5, a new formulation for Friedrichs' systems on the whole space is proposed, very similar to the Kruzhkov's definition of entropy solutions of scalar quasilinear equations 11 and to dissipative solutions introduced by Lions for incompressible Euler equations [13]. In [17], Otto extends the concept of entropy solutions to bounded domains with Dirichlet conditions, without requiring any assumption on the existence of traces of the solutions (contrary to the theory developed in [1]). We aim to do the same for linear systems. In order to verify that this original formalism contains enough information, we prove in the classical case of constant coefficients in the half-plane that this formulation leads to a well-posed theory in $L_{t, x}^{2}$ and that our formulation is equivalent to the usual strong formulation for sufficiently smooth solutions. Finally, since different theories of boundary conditions are available in the literature, we compare our formulation to other formulations on a simple example. 
This work is organized as follows. In the first section, we explain formally where the definition comes from, how this definition can be extended in order to take account of non-homogeneous boundary conditions and variable coefficients, and then we show in the case of constant coefficients when the domain is a half-plane that a $H^{1}$ solution of our weak formulation is a solution of the problem in the strong sense. Then we prove that there exists a unique solution (in the sense of our definition) in the non-characteristic setting for $H^{1}$ initial data and $L^{2}$ source term, again for constant coefficients and if the spatial domain is a half-plane (the regularity of the initial data is necessary for our proof, but in this case, the classical theory is valid for $L^{2}$ initial data and $L^{2}$ source term). Finally we compare our approach of the boundary conditions to the literature (namely to [9], $[3$ ] and [6]) and we examine these boundary conditions on the simple example of the wave equation in $1 \mathrm{D}$ with Dirichlet condition.

\section{Definition and basic properties}

\subsection{Motivations and definitions}

We are interested here in the following problem

$$
\begin{cases}\partial_{t} U+\sum_{i=1}^{n} A_{i} \partial_{x_{i}} U=f, & \text { on }(0, T) \times \Omega, \\ U(0, x)=U_{0}(x), & \text { on } \Omega \\ (A(\nu(x))-M(\nu(x))) U(t, x)=0, & \text { on }(0, T) \times \partial \Omega\end{cases}
$$

where $\Omega$ is a open domain (not necessarily bounded) of $\mathbb{R}^{n}$ with (smooth) boundary, denoted by $\partial \Omega$, $U$ is a vector of size $m, f \in L^{2}((0, T) \times \Omega)^{m}, U_{0} \in L^{2}(\Omega)^{m}$ (in the following $L^{2}((0, T) \times \Omega)^{m}$ will be simply denoted by $L_{t, x}^{2}$ and $L^{2}(\Omega)^{m}$ by $\left.L_{x}^{2}\right)$, the matrices $A_{i}$ are symmetric, $A(\nu(x))=\sum_{i=1}^{n} \nu_{i} A_{i}$ and $\nu(x)=\left(\nu_{1}, \nu_{2}, \ldots, \nu_{n}\right)$ is the exterior unit normal of $\Omega$ at the point $x \in \partial \Omega, M(\nu(x))$ is a symmetric non-negative matrix such that

$$
\operatorname{ker} A(\nu(x)) \subset \operatorname{ker} M(\nu(x))
$$

and

$$
\mathbb{R}^{m}=\operatorname{ker}(A(\nu(x))-M(\nu(x)))+\operatorname{ker}(A(\nu(x))+M(\nu(x)))
$$

The symmetry of the matrix $M$ is an important hypothesis in our approach. In the work of Friedrichs 9 ] the matrix $M$ does not need to be symmetric, but its symmetric part has to be non-negative (see section 4). In his work, Friedrichs only considers matrices $M$ such that the previous sum is direct (this hypothesis is too restrictive, especially in the case where $\operatorname{det}(A(\nu(x))=0$ ), but one can see (using lemma 1) that in the non-characteristic case $(i . e . \operatorname{det}(A(\nu(x)) \neq 0)$ the sum is always direct. In the following, the matrices $A_{i}$ are independent of the space and time variables but our formulation can be extended to matrices with variables coefficients (see remark 5 ).

Remark 1 To design a matrix $M(\nu(x))$, we could consider an orthogonal matrix $P(\nu(x))$ such that ${ }^{t} P(\nu(x)) A(\nu(x)) P(\nu(x))$ is a diagonal matrix and its diagonal terms are $\lambda_{1,1} \leq \cdots \leq \lambda_{p, p}<\lambda_{p+1, p+1}=$ $0=\cdots=\lambda_{q, q}<\cdots \leq \lambda_{m, m}$, then define

$$
M(\nu(x))={ }^{t} P(\nu(x)) \operatorname{diag}\left(\left|\lambda_{1,1}\right|, \cdots,\left|\lambda_{p, p}\right|, 0, \cdots, 0, \lambda_{q+1, q+1}, \cdots, \lambda_{m, m}\right) P(\nu(x)) .
$$

It means there is no information coming from the incoming characteristic (since we consider here an homogeneous boundary condition). The hypothesis that $M$ is symmetric still allows us to use a large class of boundary conditions coming from the theory of characteristics.

To shorten notation, we write $A_{\nu}$ instead of $A(\nu(x))$ and $M$ instead of $M(\nu(x))$.

Remark 2 Here we denote by $\langle. \mid$.$\rangle the canonical scalar product on \mathbb{R}^{m}$. 


\section{Lemma 1}

Let $A_{\nu}$ and $M$ be symmetric matrices verifying the properties (2) and (3). The following statements are true

1. We have the decomposition

$$
\mathbb{R}^{m}=\operatorname{ker} A_{\nu} \oplus\left(\left(\operatorname{ker}\left(A_{\nu}-M\right)\right) \cap \operatorname{Im} A_{\nu}\right) \oplus\left(\left(\operatorname{ker}\left(A_{\nu}+M\right)\right) \cap \operatorname{Im} A_{\nu}\right),
$$

and we denote $k=k^{0}+k_{-}+k_{+}$the decomposition of $k \in \mathbb{R}^{m}$ over this sum.

2. $M$ is positive on $\left(\operatorname{ker}\left(A_{\nu}+M\right)\right) \cap \operatorname{Im} A_{\nu}$.

3. For all $k, \kappa \in \mathbb{R}^{m},\left\langle k \mid A_{\nu} \kappa\right\rangle=\left\langle k_{-} \mid A_{\nu} \kappa_{-}\right\rangle+\left\langle k_{+} \mid A_{\nu} \kappa_{+}\right\rangle$.

4. For all $k \in \mathbb{R}^{m}, 0 \leq\left\langle k_{-} \mid M k_{-}\right\rangle \leq\langle k \mid M k\rangle$ and $0 \leq\left\langle k_{+} \mid M k_{+}\right\rangle \leq\langle k \mid M k\rangle$.

The proof of this lemma uses classical tools of linear algebra. It allows us to make some algebraic manipulations (see below) to derive the following definition

Definition 1 We say that $U \in L_{t, x}^{2}$ is a dissipative solution of the system (1) if for all $\varphi \in W_{c,+}^{1, \infty}$, the space of $W^{1, \infty}((0, T) \times \Omega)$ functions with non-negative values and compact supports (in $\left.\mathbb{R}^{n+1}\right)$, and for all $k \in \mathbb{R}^{m}$ the following inequality holds

$$
\begin{aligned}
& \int_{0}^{T} \int_{\Omega}|U(t, x)-k|^{2} \partial_{t} \varphi(t, x) \mathrm{d} x \mathrm{~d} t \\
& +\int_{0}^{T} \int_{\Omega} \sum_{i=1}^{n}\left\langle U(t, x)-k \mid A_{i}(U(t, x)-k)\right\rangle \partial_{x_{i}} \varphi(t, x) \mathrm{d} x \mathrm{~d} t \\
& +\int_{\Omega}\left|U_{0}(x)-k\right|^{2} \varphi(0, x) \mathrm{d} x+\int_{0}^{T} \int_{\Omega} 2\langle f(t, x) \mid U(t, x)-k\rangle \varphi(t, x) \mathrm{d} x \mathrm{~d} t \\
& +\int_{0}^{T} \int_{\partial \Omega}\left\langle k_{+} \mid M(\nu(x)) k_{+}\right\rangle \varphi(t, x) \mathrm{d} x \mathrm{~d} t \geq 0,
\end{aligned}
$$

where $k_{+}$stands for the projection on $\left(\operatorname{ker}\left(A_{\nu}+M\right)\right) \cap \operatorname{Im} A_{\nu}$ with respect to the decomposition of $\mathbb{R}^{m}$ presented in lemma 1 .

Remark 3 As $A_{\nu}$ and $M$ depend on $x \in \partial \Omega, k_{+}$also depends on $\partial \Omega$. In the case where $A_{\nu}$ and $M d o$ not depend on $x, k_{+}$is independent of $x \in \partial \Omega$ (see remark 8 for more details).

It is worth pointing out that the formulation does not require much regularity on the solution, in particular we do not use any traces of the solution either at the initial time or at the boundary. This formulation is inspired by the one exposed in [5] for Friedrichs' systems under constraints in the whole space. Furthermore, this definition can be used in the case of variable coefficients (see remark 5) and when the number of non-negative eigenvalues of $A_{\nu}$ is not constant along $\partial \Omega$, this is the case of variable multiplicities (as long as we can find a matrix $M$ that satisfies the hypotheses (2) and (3) and belongs to $L^{\infty}$ ), these cases are of deep interest for applications (the condition of constant multiplicities is difficult to insure for a general domain $\Omega$, in particular for a bounded domain $\partial \Omega$ ) and that is still an active domain of research (see for example [16]).

We call this formulation dissipative since taking $k=0$ and $\varphi(t, x)=T-t$ in (4), we get the $L^{2}$-norm of a solution is non-increasing

$$
\int_{0}^{T} \int_{\Omega}|U|^{2}(t, x) \mathrm{d} t \mathrm{~d} x \leq T \int_{\Omega}\left|U_{0}\right|^{2}(x) \mathrm{d} x .
$$

This possible loss of energy of the solution allows us to call the solution dissipative (as P-L. Lions did for Euler equations in [13]). The word dissipative also refers in the literature to special kind of boundary conditions, our boundary condition can be called dissipative in that sense (see section 4). 
Let us briefly explain where this formulation comes from. If we assume that the solution $U$ is regular and we use the fact that the derivatives of $k$ cancel, we go from (1) to

$$
\begin{aligned}
& \int_{0}^{T} \int_{\Omega}|U(t, x)-k|^{2} \partial_{t} \varphi(t, x) d x d t+\int_{0}^{T} \int_{\Omega} \sum_{i=1}^{n}\left\langle U(t, x)-k \mid A_{i}(U(t, x)-k)\right\rangle \partial_{x_{i}} \varphi(t, x) \mathrm{d} x \mathrm{~d} t \\
& +\int_{0}^{T} \int_{\Omega} 2\langle f(t, x) \mid U(t, x)-k\rangle \varphi(t, x) \mathrm{d} x \mathrm{~d} t-\int_{0}^{T} \int_{\partial \Omega}\left\langle U(t, x)-k \mid A_{\nu}(U(t, x)-k)\right\rangle \varphi(t, x) \mathrm{d} \sigma(x) \mathrm{d} t \\
& +\int_{\Omega}\left|U_{0}(x)-k\right|^{2} \varphi(0, x) \mathrm{d} x-\int_{\Omega}|U(T, x)-k|^{2} \varphi(T, x) \mathrm{d} x=0,
\end{aligned}
$$

by taking the scalar product with $U-k$ and then integrating by parts. Then we write the decomposition of $k=k^{0}+k_{-}+k_{+}$and observe that since $U \in \operatorname{ker}\left(A_{\nu}-M\right)$ at the boundary we know that $U_{+}=0$ and it follows that

$$
\begin{aligned}
& \int_{0}^{T} \int_{\partial \Omega}\left\langle U(t, x)-k \mid A_{\nu}(U(t, x)-k)\right\rangle \varphi(t, x) \mathrm{d} \sigma(x) \mathrm{d} t \\
= & \int_{0}^{T} \int_{\partial \Omega}\left\langle U(t, x)-k_{-} \mid A_{\nu}\left(U(t, x)-k_{-}\right)\right\rangle \varphi(t, x) \mathrm{d} \sigma(x) \mathrm{d} t+\int_{0}^{T} \int_{\partial \Omega}\left\langle k_{+} \mid A_{\nu} k_{+}\right\rangle \varphi(t, x) \mathrm{d} \sigma(x) \mathrm{d} t \\
= & \int_{0}^{T} \int_{\partial \Omega}\left\langle U(t, x)-k_{-} \mid M\left(U(t, x)-k_{-}\right)\right\rangle \varphi(t, x) \mathrm{d} \sigma(x) \mathrm{d} t-\int_{0}^{T} \int_{\partial \Omega}\left\langle k_{+} \mid M k_{+}\right\rangle \varphi(t, x) \mathrm{d} \sigma(x) \mathrm{d} t,
\end{aligned}
$$

thanks to lemma 1 . Consequently,

$$
\begin{aligned}
& \quad \int_{0}^{T} \int_{\Omega}|U(t, x)-k|^{2} \partial_{t} \varphi(t, x) \mathrm{d} x \mathrm{~d} t+\int_{0}^{T} \int_{\Omega} \sum_{i=1}^{n}\left\langle U(t, x)-k \mid A_{i}(U(t, x)-k)\right\rangle \partial_{x_{i}} \varphi(t, x) \mathrm{d} x \mathrm{~d} t \\
& \quad+\int_{0}^{T} \int_{\Omega} 2\langle f(t, x) \mid U(t, x)-k\rangle \varphi(t, x) \mathrm{d} x \mathrm{~d} t+\int_{\Omega}\left|U_{0}(x)-k\right|^{2} \varphi(0, x) \mathrm{d} x \\
& \quad+\int_{0}^{T} \int_{\partial \Omega}\left\langle k_{+} \mid M k_{+}\right\rangle \varphi(t, x) \mathrm{d} \sigma(x) \mathrm{d} t \\
& =\int_{0}^{T} \int_{\partial \Omega}\left\langle U(t, x)-k_{-} \mid M\left(U(t, x)-k_{-}\right)\right\rangle \varphi(t, x) \mathrm{d} \sigma(x)+\int_{\Omega}|U(T, x)-k|^{2} \varphi(T, x) \mathrm{d} x \mathrm{~d} t \\
& \geq \quad 0 .
\end{aligned}
$$

Remark 4 Here we explain how to take account of an inhomogeneous boundary condition of the form

$$
\left(A_{\nu}-M\right)\left(U(t, x)-U_{b}(t, x)\right)=0, \quad \text { on }(0, T) \times \partial \Omega,
$$

where $U_{b} \in L^{2}((0, T) \times \partial \Omega)^{m}$. From the previous formal calculations, we want to eliminate the trace of $U$ in the term

$$
\int_{0}^{T} \int_{\partial \Omega}\left\langle U(t, x)-k \mid A_{\nu}(U(t, x)-k)\right\rangle \varphi(t, x) \mathrm{d} \sigma(x) \mathrm{d} t .
$$

Since we know that, formally and using lemma 1 .

$$
\begin{aligned}
& \left\langle U-k \mid A_{\nu}(U-k)\right\rangle \\
= & \left\langle U_{b}-k \mid A_{\nu}\left(U_{b}-k\right)\right\rangle+\left\langle U-U_{b} \mid A_{\nu}\left(U-U_{b}\right)\right\rangle+2\left\langle U-U_{b} \mid A_{\nu}\left(U_{b}-k\right)\right\rangle \\
= & \left\langle\left(U_{b}-k\right)_{-} \mid M\left(U_{b}-k\right)_{-}\right\rangle-\left\langle\left(U_{b}-k\right)_{+} \mid M\left(U_{b}-k\right)_{+}\right\rangle \\
& +\left\langle U-U_{b} \mid M\left(U-U_{b}\right)\right\rangle+2\left\langle\left(U-U_{b}\right) \mid M\left(U_{b}-k\right)_{-}\right\rangle \\
\geq & -\left\langle\left(U_{b}-k\right)_{+} \mid M\left(U_{b}-k\right)_{+}\right\rangle,
\end{aligned}
$$

we just replace the term

by

$$
\int_{0}^{T} \int_{\partial \Omega}\left\langle k_{+} \mid M k_{+}\right\rangle \varphi(t, x) \mathrm{d} t \mathrm{~d} \sigma(x)
$$

$$
\int_{0}^{T} \int_{\partial \Omega}\left\langle\left(k-U_{b}\right)_{+} \mid M\left(k-U_{b}\right)_{+}\right\rangle \varphi(t, x) \mathrm{d} t \mathrm{~d} \sigma(x),
$$

in the formulation. 
Remark 5 We also can write a definition of solution for symmetric matrices $A_{i}$ that belong to the space $W^{1, \infty}((0, T) \times \Omega)^{m \times m}$ (assuming that one can find a matrix $M$ that satisfies the conditions (2) and (3) and belongs to $\left.L^{\infty}((0, T) \times \partial \Omega)^{m \times m}\right)$. One just need to add the term

$$
\int_{0}^{T} \int_{\Omega} \sum_{i=1}^{n}\left\langle U(t, x)-k \mid \partial_{x_{i}}\left[A_{i}\right](U(t, x)-k)\right\rangle \varphi(t, x) \mathrm{d} x \mathrm{~d} t,
$$

to the left-hand side of the inequality (4).

Remark 6 One can see in the functions parametrized by $k \in \mathbb{R}^{m}$

$$
F(U)=|U-k|^{2}
$$

the analogue of the entropy functions of Kružkov [11] in the scalar case of quasilinear initial value problems.

Example 1 In the scalar case $(m=1)$, the theory of initial boundary value problems is well known ([1], [17]). Using our theory, we see that if $A_{\nu} \geq 0$ then $M=A_{\nu}$ is the only choice for the boundary matrix and it leads to the fact that there is no condition at that point (this corresponds exactly to the case when the BLN condition [1] is automatically verified whatever the trace is at that point). In the case $A_{\nu}<0$, the choice for $M$ is $-A_{\nu}$, it implies (at least formally) that $U=0$ at this point of the boundary (again this corresponds to the BLN condition).

\subsection{What is the information contained in our formulation?}

Our goal is here to show that dissipative solutions (which satisfy inequalities) satisfy the original equation, initial condition and boundary condition in a weak sense (that are equalities). For technical reasons we will nevertheless make the hypothesis that the spatial domain is a half-plane for the study of the boundary condition.

First, we are going to show that if $U \in L_{t, x}^{2}$ is a solution of (1) in the sense of definition 1 then $U$ is a solution of the equation

$$
\partial_{t} U+\sum_{i=1}^{n} A_{i} \partial_{x_{i}} U=f
$$

in the sense of the distributions.

\section{Lemma 2}

A solution $U$ in the sense of definition 1 verifies

$$
\partial_{t} U+\sum_{i=1}^{n} A_{i} \partial_{x_{i}} U=0, \quad \text { in } \mathcal{D}^{\prime}((0, T) \times \Omega) .
$$

Proof: Let $\varphi$ be a function of $\mathcal{D}((0, T) \times \Omega)$ with non-negative values. Then, from definition 1 , we know that

$$
\begin{aligned}
\int_{0}^{T} \int_{\Omega}|U(t, x)-k|^{2} \partial_{t} \varphi(t, x) \mathrm{d} x \mathrm{~d} t \\
\quad+\int_{0}^{T} \int_{\Omega} \sum_{i=1}^{n}\left\langle U(t, x)-k \mid A_{i}(U(t, x)-k)\right\rangle \partial_{x_{i}} \varphi(t, x) \mathrm{d} x \mathrm{~d} t \geq 0 .
\end{aligned}
$$

Expanding the terms $|U(t, x)-k|^{2}$ and $\left\langle U(t, x)-k \mid A_{i}(U(t, x)-k)\right\rangle$ and using the fact that the partial derivatives of $k$ vanish leads us to the following inequality

$$
\begin{aligned}
\int_{0}^{T} \int_{\Omega} & {\left[\left(|U(t, x)|^{2}-2\langle k \mid U(t, x)\rangle\right) \partial_{t} \varphi(t, x)\right.} \\
+ & \left.\sum_{i=1}^{n}\left(\left\langle U(t, x) \mid A_{i} U(t, x)\right\rangle-2\left\langle U(t, x) \mid A_{i} k\right\rangle\right) \partial_{x_{i}} \varphi(t, x)\right] \mathrm{d} x \mathrm{~d} t \geq 0 .
\end{aligned}
$$

Since $k$ is independent of $t$ and $x$, we rewrite the previous inequality as

$$
\begin{array}{r}
\int_{0}^{T} \int_{\Omega}\left[|U(t, x)|^{2} \partial_{t} \varphi(t, x)+\sum_{i=1}^{n}\left\langle U(t, x) \mid A_{i} U(t, x)\right\rangle \partial_{x_{i}} \varphi(t, x)\right] \mathrm{d} x \mathrm{~d} t \geq \\
2\left\langle k \mid \int_{0}^{T} \int_{\Omega} U(t, x) \partial_{t} \varphi(t, x)+\sum_{i=1}^{n} A_{i} U(t, x) \partial_{x_{i}} \varphi(t, x) \mathrm{d} x \mathrm{~d} t\right\rangle .
\end{array}
$$


The left-hand side of the inequality is independent of $k$, thus the previous inequality implies that

$$
\int_{0}^{T} \int_{\Omega} U(t, x) \partial_{t} \varphi(t, x)+\sum_{i=1}^{n} A_{i} U(t, x) \partial_{x_{i}} \varphi(t, x) \mathrm{d} x \mathrm{~d} t=0 .
$$

It remains to show that equation $(6)$ is true for all $\varphi \in \mathcal{D}((0, T) \times \Omega)$. Let $\varphi$ be a function in $\mathcal{D}((0, T) \times \Omega)$, then $\varphi=\varphi^{+}-\varphi^{-}$where $\varphi^{+}$(resp. $\left.\varphi^{-}\right)$is the positive part (resp. negative part) of $\varphi$ and $\varphi^{+}$(resp. $\varphi^{-}$) is an element of $W_{c,+}^{1, \infty}((0, T) \times \Omega)$. Equation [6] is true for $\varphi^{+}\left(\right.$resp. $\left.\varphi^{-}\right)$and thus the lemma is proven.

Remark 7 The previous and following lemmas are also true in the case of variable coefficients and when $\Omega$ is a general domain with smooth boundary.

Now, we focus our attention on the initial condition and the boundary condition.

\section{Lemma 3}

Let $\xi \in W_{c,+}^{1, \infty}(\Omega)$. We have

$$
\lim _{\tau \rightarrow 0} \lim _{\alpha \rightarrow 0} \frac{1}{\alpha} \int_{\tau-\alpha}^{\tau} \int_{\Omega}\left|U(t, x)-U_{0}(x)\right|^{2} \xi(x) \mathrm{d} x \mathrm{~d} t=0 .
$$

Proof: For convenience, we take $f=0$. The same conclusion can be drawn for a general $f \in L_{t, x}^{2}$. Let $\xi \in W_{c,+}^{1, \infty}(\Omega)$ and $\rho_{n}^{\epsilon}$ be the classical positive $n$-dimensional mollifier with $\epsilon>0$. Let $\alpha>0$ and $\tau \in(\alpha, T)$. We define the function $\varphi:[0, T] \times \Omega \times \Omega \rightarrow \mathbb{R}^{+}$by

$$
\varphi(t, x, y)=\xi(x) \eta_{\alpha}(t) \rho_{n}^{\epsilon}(x-y)
$$

where $\eta_{\alpha}(t)=\max (0, \min (1,(\tau-t) / \alpha))$. Observe that $\eta_{\alpha}^{\prime}(t) \leq 0$ and $\eta_{\alpha}^{\prime} \underset{\alpha \rightarrow 0^{+}}{\longrightarrow}-\delta_{\tau}$ in the distributional sense. Using the Cauchy-Schwarz inequality, we have

$$
\begin{aligned}
& \left|U(t, x)-U_{0}(y)\right|^{2} \\
= & \left|U(t, x)-U_{0}(x)\right|^{2}+\left|U_{0}(x)-U_{0}(y)\right|^{2}+2\left\langle U(t, x)-U_{0}(x) \mid U_{0}(x)-U_{0}(y)\right\rangle \\
\geq & \left|U(t, x)-U_{0}(x)\right|^{2}+\left|U_{0}(x)-U_{0}(y)\right|^{2}-2\left|U(t, x)-U_{0}(x)\right|\left|U_{0}(x)-U_{0}(y)\right| .
\end{aligned}
$$

From the inequality $\partial_{t} \varphi \leq 0$, we get for almost every $(t, x, y)$,

$$
\begin{aligned}
& \left|U(t, x)-U_{0}(y)\right|^{2} \partial_{t} \varphi(t, x, y) \\
\leq & \left|U(t, x)-U_{0}(x)\right|^{2} \partial_{t} \varphi(t, x, y)+\left|U_{0}(x)-U_{0}(y)\right|^{2} \partial_{t} \varphi(t, x, y) \\
& -2\left|U(t, x)-U_{0}(x)\right|\left|U_{0}(x)-U_{0}(y)\right| \partial_{t} \varphi(t, x, y) .
\end{aligned}
$$

We integrate this inequality over $[0, T] \times \mathbb{R}^{n} \times \mathbb{R}^{n}$ (we extend $U$ and $U_{0}$ by zero outside of $\Omega$ ) to get, thanks to Fubini's theorem,

$$
\begin{aligned}
& \int_{0}^{T} \int_{\mathbb{R}^{n}} \int_{\mathbb{R}^{n}}\left|U(t, x)-U_{0}(y)\right|^{2} \partial_{t} \varphi(t, x, y) \mathrm{d} x \mathrm{~d} y \mathrm{~d} t \\
\leq & \int_{0}^{T} \int_{\mathbb{R}^{n}} \int_{\mathbb{R}^{n}}\left|U(t, x)-U_{0}(x)\right|^{2} \partial_{t} \varphi(t, x, y) \mathrm{d} x \mathrm{~d} y \mathrm{~d} t-\int_{\mathbb{R}^{n}} \int_{\mathbb{R}^{n}}\left|U_{0}(x)-U_{0}(y)\right|^{2} \xi(x) \rho_{n}^{\epsilon}(x-y) \mathrm{d} x \mathrm{~d} y \\
& -2 \int_{\mathbb{R}^{n}} \int_{\mathbb{R}^{n}}\left|U_{0}(x)-U_{0}(y)\right| \xi(x) \rho_{n}^{\epsilon}(x-y) \int_{0}^{T}\left|U(t, x)-U_{0}(x)\right| \eta_{\alpha}^{\prime}(t) \mathrm{d} t \mathrm{~d} x \mathrm{~d} y .
\end{aligned}
$$

We take $k=U_{0}(y)$ and use the above-defined test function $\varphi(t, x, y)$ in the definition of a dissipative solution and integrate with respect to $y$ to obtain

$$
\begin{aligned}
& \int_{0}^{T} \int_{\mathbb{R}^{n}} \int_{\mathbb{R}^{n}}\left|U(t, x)-U_{0}(y)\right|^{2} \partial_{t} \varphi(t, x, y) \mathrm{d} x \mathrm{~d} y \mathrm{~d} t \\
\geq & -\int_{0}^{T} \mathcal{R}_{\alpha, \epsilon}(t) \mathrm{d} t-\int_{\mathbb{R}^{n}} \int_{\mathbb{R}^{n}}\left|U_{0}(x)-U_{0}(y)\right|^{2} \xi(x) \rho_{n}^{\epsilon}(x-y) \mathrm{d} x \mathrm{~d} y
\end{aligned}
$$

where $\mathcal{R}_{\alpha, \epsilon}$ belongs to $L^{1}(0, T)$ and is for almost every time $t$ defined by

$$
\begin{aligned}
\mathcal{R}_{\alpha, \epsilon}(t)= & \int_{\mathbb{R}^{n}} \int_{\mathbb{R}^{n}} \sum_{i=1}^{n}\left\langle U(t, x)-U_{0}(y) \mid A_{i}\left(U(t, x)-U_{0}(y)\right)\right\rangle \eta_{\alpha}(t) \partial_{x_{i}}\left(\xi(x) \rho_{n}^{\epsilon}(x-y)\right) \mathrm{d} x \mathrm{~d} y \\
& +\int_{\mathbb{R}^{n}} \int_{\partial \Omega}\left\langle U_{0}(y)_{+} \mid M U_{0}(y)_{+}\right\rangle \xi(x) \eta_{\alpha}(t) \rho_{n}^{\epsilon}(x-y) \mathrm{d} \sigma(x) \mathrm{d} y .
\end{aligned}
$$


Using these two inequalities, we get

$$
\begin{aligned}
-\int_{0}^{T} \mathcal{R}_{\alpha, \epsilon}(t) \mathrm{d} t \leq & \int_{0}^{T} \int_{\mathbb{R}^{n}} \int_{\mathbb{R}^{n}}\left|U(t, x)-U_{0}(x)\right|^{2} \partial_{t} \varphi(t, x, y) \mathrm{d} x \mathrm{~d} y \mathrm{~d} t \\
& -2 \int_{\mathbb{R}^{n}} \int_{\mathbb{R}^{n}}\left|U_{0}(x)-U_{0}(y)\right| \xi(x) \rho_{n}^{\epsilon}(x-y) \int_{0}^{T}\left|U(t, x)-U_{0}(x)\right| \eta_{\alpha}^{\prime}(t) \mathrm{d} t \mathrm{~d} x \mathrm{~d} y .
\end{aligned}
$$

Using the fact that for $(a, b) \in \mathbb{R}^{2}, a b \leq b^{2}+\frac{a^{2}}{4}$ and, since $\partial_{t} \varphi \leq 0$,

$$
\begin{aligned}
& -\left|U_{0}(x)-U_{0}(y)\right|\left|U(t, x)-U_{0}(x)\right| \xi(x) \rho_{n}^{\epsilon}(x-y) \eta_{\alpha}^{\prime}(t) \\
= & \left|U_{0}(x)-U_{0}(y)\right|\left|U(t, x)-U_{0}(x)\right|\left|\partial_{t} \varphi(t, x, y)\right| \\
\leq & \left|U_{0}(x)-U_{0}(y)\right|^{2} \xi(x) \rho_{n}^{\epsilon}(x-y)\left|\eta_{\alpha}^{\prime}(t)\right|-\frac{\left|U(t, x)-U_{0}(x)\right|^{2}}{4} \partial_{t} \varphi(t, x, y) .
\end{aligned}
$$

By integration of this inequality over $[0, T] \times \mathbb{R}^{n} \times \mathbb{R}^{n}$, it follows

$$
\begin{aligned}
-\int_{0}^{T} \mathcal{R}_{\alpha, \epsilon}(t) \mathrm{d} t \leq & \frac{3}{4} \int_{0}^{T} \int_{\mathbb{R}^{n}} \int_{\mathbb{R}^{n}}\left|U(t, x)-U_{0}(x)\right|^{2} \partial_{t} \varphi(t, x, y) \mathrm{d} x \mathrm{~d} y \mathrm{~d} t \\
& +\int_{\mathbb{R}^{n}} \int_{\mathbb{R}^{n}}\left|U_{0}(x)-U_{0}(y)\right|^{2} \xi(x) \rho_{n}^{\epsilon}(x-y) \mathrm{d} x \mathrm{~d} y .
\end{aligned}
$$

We are going to send $\alpha$ to 0 . By the definition of $\eta_{\alpha}$ (and since $\eta_{0}=\mathbb{1}_{[0, \tau]}$ ) and equation (7), we have

$$
\begin{aligned}
& \left|\int_{0}^{T}\left[\mathcal{R}_{\alpha, \epsilon}(t)-\mathcal{R}_{0, \epsilon}(t)\right] \mathrm{d} t\right| \\
\leq & \int_{\tau-\alpha}^{\tau} \int_{\mathbb{R}^{n}} \int_{\mathbb{R}^{n}} \sum_{i=1}^{n}\left|\left\langle U(t, x)-U_{0}(y) \mid A_{i}\left(U(t, x)-U_{0}(y)\right)\right\rangle \partial_{x_{i}} \varphi(t, x, y)\right| \mathrm{d} x \mathrm{~d} y \mathrm{~d} t \\
& +2 \int_{\tau-\alpha}^{\tau} \int_{\mathbb{R}^{n}} \int_{\partial \Omega}\left\langle U_{0}(y)_{+} \mid M U_{0}(y)_{+}\right\rangle \varphi(t, x, y) \mathrm{d} \sigma(x) \mathrm{d} y \mathrm{~d} t .
\end{aligned}
$$

We now use the fact that, if $k=k^{0}+k_{-}+k_{+}$with $k_{ \pm} \in \operatorname{ker}(A \pm M) \cap \operatorname{Im} A$ and $k^{0} \in \operatorname{ker} A$, then $\left\langle k_{-} \mid M k_{+}\right\rangle=0$ and consequently $0 \leq\left\langle k_{+} \mid M k_{+}\right\rangle \leq\langle k \mid M k\rangle$. Therefore we know that the right-hand side of the previous inequality belongs to $L^{1}(0, T)$, so the dominated convergence theorem implies that

$$
\lim _{\alpha \rightarrow 0} \int_{0}^{T} \mathcal{R}_{\alpha, \epsilon}(t) \mathrm{d} t=\int_{0}^{T} \mathcal{R}_{0, \epsilon}(t) \mathrm{d} t .
$$

It follows that, when $\alpha \rightarrow 0$,

$$
\begin{aligned}
& \lim _{\alpha \rightarrow 0} \frac{1}{\alpha} \int_{\tau-\alpha}^{\tau} \int_{\mathbb{R}^{n}} \int_{\mathbb{R}^{n}}\left|U(t, x)-U_{0}(x)\right|^{2} \xi(x) \rho_{n}^{\epsilon}(x-y) \mathrm{d} x \mathrm{~d} y \mathrm{~d} t \\
\leq & \frac{4}{3} \int_{0}^{T} \mathcal{R}_{0, \epsilon}(t) \mathrm{d} t+4 \int_{\mathbb{R}^{n}} \int_{\mathbb{R}^{n}}\left|U_{0}(x)-U_{0}(y)\right|^{2} \xi(x) \rho_{n}^{\epsilon}(x-y) \mathrm{d} x \mathrm{~d} y .
\end{aligned}
$$

But, by Fubini's theorem, we remark that for every $\epsilon>0$,

$$
\int_{\mathbb{R}^{n}} \int_{\mathbb{R}^{n}}\left|U(t, x)-U_{0}(x)\right|^{2} \xi(x) \rho_{n}^{\epsilon}(x-y) \mathrm{d} x \mathrm{~d} y=\int_{\mathbb{R}^{n}}\left|U(t, x)-U_{0}(x)\right|^{2} \xi(x) \mathrm{d} x .
$$

Then, we let $\tau$ going to 0 in the last inequality, and we use that for all $\epsilon$, we know that

$$
\begin{aligned}
\left|\int_{0}^{T} \mathcal{R}_{0, \epsilon}(t) \mathrm{d} t\right| \leq & \int_{0}^{\tau} \int_{\mathbb{R}^{n}} \int_{\mathbb{R}^{n}} \sum_{i=1}^{n} C_{i}\left(\left|U_{0}\right|^{2}(x)+\left|U_{0}\right|^{2}(y)\right) \partial_{x_{i}}\left[\xi(x) \rho_{n}^{\epsilon}(x-y)\right] \mathrm{d} x \mathrm{~d} y \mathrm{~d} t \\
& +\int_{0}^{\tau} \int_{\mathbb{R}^{n}} \int_{\partial \Omega} C\left|U_{0}\right|^{2}(y)\|\xi\|_{\infty} \mathrm{d} \sigma(x) \mathrm{d} y \mathrm{~d} t
\end{aligned}
$$

where $C_{i}$ and $C$ respectively depend on $A_{i}$ and $M$. By the dominated convergence theorem, we get

$$
\begin{aligned}
\lim _{T \rightarrow 0} \lim _{\alpha \rightarrow 0} \frac{1}{\alpha} \int_{\tau-\alpha}^{\tau} \int_{\Omega}\left|U(t, x)-U_{0}(x)\right|^{2} \xi(x) \mathrm{d} x \mathrm{~d} t & =\lim _{T \rightarrow 0} \lim _{\alpha \rightarrow 0} \frac{1}{\alpha} \int_{\tau-\alpha}^{\tau} \int_{\mathbb{R}^{n}}\left|U(t, x)-U_{0}(x)\right|^{2} \xi(x) \mathrm{d} x \mathrm{~d} t \\
& \leq 4 \int_{\mathbb{R}^{n}} \int_{\mathbb{R}^{n}}\left|U_{0}(x)-U_{0}(y)\right|^{2} \xi(x) \rho_{n}^{\epsilon}(x-y) \mathrm{d} x \mathrm{~d} y .
\end{aligned}
$$

The left-hand side is independent of $\epsilon$ and the right-hand side goes to 0 as $\epsilon$ goes to 0 . 
We now discuss the boundary condition in the special case of the $n$-dimensional half-place

$$
\Omega=\mathbb{R}_{-}^{n}=\left\{\left(x_{1}, x_{2}, \cdots, x_{n}\right)=\left(x^{\prime}, x_{n}\right), x_{n}<0\right\} .
$$

In the following, we assume that $\Omega=\mathbb{R}_{-}^{n}$ and that the matrices $A_{i}$ and $M$ are independent of the space and time variables (see remark 8 for more details).

\section{Lemma 4}

Assume $\Omega$ is a half-space (8). If $U \in L_{t, x}^{2}$ is a solution of (1) in $(0, T) \times \mathbb{R}_{-}^{n}$ in the sense of definition 1 , then $U$ satisfies the following condition at the boundary

$$
\lim _{\epsilon \rightarrow 0} \frac{1}{\epsilon} \int_{\mathbb{R}^{n-1}} \int_{-\epsilon}^{0} \int_{0}^{T} U(t, x)_{+} \psi(t) \lambda\left(x^{\prime}\right) \mathrm{d} t \mathrm{~d} x_{n} \mathrm{~d} x^{\prime}=0,
$$

for every $\psi \in W^{1, \infty}((0, T))$ and $\lambda \in W^{1, \infty}\left(\mathbb{R}^{n-1}\right)$ with compact supports (resp. in $\mathbb{R}$ and in $\mathbb{R}^{n-1}$ ).

Proof: Using the fact that any function of $W^{1, \infty}$ with compact support is the sum of its positive part and the opposite of its negative part (that belong to $\left.W_{c,+}^{1, \infty}\right)$, we may suppose that $\psi \in W_{c,+}^{1, \infty}((0, T))$ and $\lambda \in W_{c,+}^{1, \infty}\left(\mathbb{R}^{n-1}\right)$. We take in the formulation of a solution in definition 1 the function $\phi(x, t)=$ $a_{\epsilon}\left(x_{n}\right) \psi(t) \lambda\left(x^{\prime}\right)$ where $a_{\epsilon}\left(x_{n}\right)=\max \left(0,\left(x_{n}+\epsilon\right) / \epsilon\right)$. We get

$$
\begin{aligned}
& \int_{\mathbb{R}^{n-1}} \int_{-\epsilon}^{0} \int_{0}^{T}|U(t, x)-k|^{2} a_{\epsilon}\left(x_{n}\right) \lambda\left(x^{\prime}\right) \psi^{\prime}(t) \mathrm{d} t \mathrm{~d} x_{n} \mathrm{~d} x^{\prime} \\
& +\frac{1}{\epsilon} \int_{\mathbb{R}^{n-1}} \int_{-\epsilon}^{0} \int_{0}^{T}\left\langle U-k \mid A_{n}(U-k)\right\rangle \psi(t) \lambda\left(x^{\prime}\right) \mathrm{d} t \mathrm{~d} x_{n} \mathrm{~d} x^{\prime} \\
& +\int_{\mathbb{R}^{n-1}} \int_{-\epsilon}^{0} \int_{0}^{T} \sum_{i=1}^{n-1}\left\langle U-k \mid A_{i}(U-k)\right\rangle \psi(t) a_{\epsilon}\left(x_{n}\right) \partial_{x_{i}} \lambda\left(x^{\prime}\right) \mathrm{d} t \mathrm{~d} x_{n} \mathrm{~d} x^{\prime} \\
& +\int_{0}^{T} \int_{\mathbb{R}^{n-1}}\left\langle k_{+} \mid M k_{+}\right\rangle \psi(t) \lambda\left(x^{\prime}\right) \mathrm{d} t \mathrm{~d} x^{\prime} \geq 0,
\end{aligned}
$$

then we use the fact that for all vector $v \in \mathbb{R}^{n}$ we know that

$$
\langle v \mid A v\rangle=\left\langle v_{-} \mid M v_{-}\right\rangle-\left\langle v_{+} \mid M v_{+}\right\rangle,
$$

and we apply this identity in the second integral to get

$$
\begin{aligned}
& \int_{\Omega_{\epsilon}} \int_{0}^{T}|U(t, x)-k|^{2} a_{\epsilon}(x) \psi^{\prime}(t) \lambda\left(x^{\prime}\right) \mathrm{d} t \mathrm{~d} x_{n} \mathrm{~d} x^{\prime} \\
& +\int_{\Omega_{\epsilon}} \int_{0}^{T} \sum_{i=1}^{n-1}\left\langle U-k \mid A_{i}(U-k)\right\rangle \psi(t) a_{\epsilon}\left(x_{n}\right) \partial_{x_{i}} \lambda\left(x^{\prime}\right) \mathrm{d} t \mathrm{~d} x_{n} \mathrm{~d} x^{\prime} \\
& +\frac{1}{\epsilon} \int_{\Omega_{\epsilon}} \int_{0}^{T}\left\langle(U(t, x)-k)_{-} \mid M(U(t, x)-k)_{-}\right\rangle \psi(t) \lambda\left(x^{\prime}\right) \mathrm{d} t \mathrm{~d} x_{n} \mathrm{~d} x^{\prime} \\
& -\frac{1}{\epsilon} \int_{\Omega_{\epsilon}} \int_{0}^{T}\left\langle(U(t, x)-k)_{+} \mid M(U(t, x)-k)_{+}\right\rangle \psi(t) \lambda\left(x^{\prime}\right) \mathrm{d} t \mathrm{~d} x_{n} \mathrm{~d} x^{\prime} \\
& +\int_{0}^{T} \int_{\mathbb{R}^{n-1}}\left\langle k_{+} \mid M k_{+}\right\rangle \psi(t) \lambda\left(x^{\prime}\right) \mathrm{d} t \mathrm{~d} x^{\prime} \geq 0,
\end{aligned}
$$

where $\Omega_{\epsilon}=\mathbb{R}^{n-1} \times(-\epsilon, 0)$. The linearity of the projector and the fact that $k_{+}$and $M$ are independent of $x$ leads us to

$$
\begin{aligned}
& \int_{\Omega_{\epsilon}} \int_{0}^{T}|U(t, x)-k|^{2} a_{\epsilon}(x) \psi^{\prime}(t) \lambda\left(x^{\prime}\right) \mathrm{d} t \mathrm{~d} x_{n} \mathrm{~d} x^{\prime} \\
& +\frac{1}{\epsilon} \int_{\Omega_{\epsilon}} \int_{0}^{T}\left\langle(U(t, x)-k)_{-} \mid M(U(t, x)-k)_{-}\right\rangle \psi(t) \lambda\left(x^{\prime}\right) \mathrm{d} t \mathrm{~d} x_{n} \mathrm{~d} x^{\prime} \\
& +\int_{\Omega_{\epsilon}} \int_{0}^{T} \sum_{i=1}^{n-1}\left\langle U-k \mid A_{i}(U-k)\right\rangle a_{\epsilon}(x) \psi(t) \partial_{x_{i}} \lambda\left(x^{\prime}\right) \mathrm{d} t \mathrm{~d} x_{n} \mathrm{~d} x^{\prime} \geq \\
& \frac{1}{\epsilon} \int_{\Omega_{\epsilon}} \int_{0}^{T}\left\langle U(t, x)_{+} \mid M U(t, x)_{+}\right\rangle \psi(t) \lambda\left(x^{\prime}\right) \mathrm{d} t \mathrm{~d} x_{n} \mathrm{~d} x^{\prime}-\frac{2}{\epsilon} \int_{\Omega_{\epsilon}} \int_{0}^{T}\left\langle U(t, x)_{+} \mid M k_{+}\right\rangle \psi(t) \lambda\left(x^{\prime}\right) \mathrm{d} t \mathrm{~d} x_{n} \mathrm{~d} x^{\prime} .
\end{aligned}
$$


Now, we set $k=-\alpha k_{0}$ where $k_{0}=\frac{1}{\epsilon} \int_{\Omega_{\epsilon}} \int_{0}^{T} U(t, x)_{+} \psi(t) \lambda\left(x^{\prime}\right) \mathrm{d} t \mathrm{~d} x_{n} \mathrm{~d} x^{\prime}$ and $\alpha \in \mathbb{R}$ is to be chosen later. Observe that $k_{0} \in \operatorname{ker}(A+M) \cap \operatorname{Im} A$ and consequently $k_{-}=0$, using that $k$ in the previous inequality we obtain

$$
\begin{aligned}
2 \alpha\left\langle k_{0} \mid M k_{0}\right\rangle \leq & 2 \alpha^{2} C_{1} \int_{-\epsilon}^{0} \int_{\mathbb{R}^{n-1} \cap \operatorname{supp} \lambda} \int_{0}^{T}\left|k_{0}\right|^{2} a_{\epsilon}(x) \mathrm{d} t \mathrm{~d} x_{n} \mathrm{~d} x^{\prime} \\
& +2 C_{2} \int_{-\epsilon}^{0} \int_{\mathbb{R}^{n-1} \cap \operatorname{supp} \lambda} \int_{0}^{T}|U(t, x)|^{2} a_{\epsilon}(x) \mathrm{d} t \mathrm{~d} x_{n} \mathrm{~d} x^{\prime} \\
& +\frac{1}{\epsilon} \int_{\Omega_{\epsilon}} \int_{0}^{T}\left\langle U(t, x)_{-} \mid M U(t, x)_{-}\right\rangle \psi(t) \lambda\left(x^{\prime}\right) d t d x_{n} \mathrm{~d} x^{\prime},
\end{aligned}
$$

where $C_{1}$ and $C_{2}$ are positive number depending on $A_{i}, \lambda$ and $\psi$. We now choose $\alpha$ to be equal to

$$
\alpha=\frac{\left\langle k_{0} \mid M k_{0}\right\rangle}{2 C_{1} \int_{-\epsilon}^{0} \int_{\mathbb{R}^{n-1} \cap \operatorname{supp} \lambda} \int_{0}^{T}\left|k_{0}\right|^{2} a_{\epsilon}(x) \mathrm{d} t \mathrm{~d} x_{n}},
$$

and consequently we get that

$$
2 \alpha\left\langle k_{0} \mid M k_{0}\right\rangle-2 \alpha^{2} C_{1} \int_{-\epsilon}^{0} \int_{\mathbb{R}^{n-1} \cap \operatorname{supp} \lambda} \int_{0}^{T}\left|k_{0}\right|^{2} a_{\epsilon}(x) \mathrm{d} t \mathrm{~d} x_{n} \mathrm{~d} x^{\prime}=\alpha\left\langle k_{0} \mid M k_{0}\right\rangle,
$$

and we obtain

$$
\begin{aligned}
\alpha\left\langle k_{0} \mid M k_{0}\right\rangle \leq & 2 C_{2} \int_{-\epsilon}^{0} \int_{\mathbb{R}^{n-1} \cap \operatorname{supp} \lambda} \int_{0}^{T}|U(t, x)|^{2} a_{\epsilon}(x) \mathrm{d} t \mathrm{~d} x_{n} \mathrm{~d} x^{\prime} \\
& +\frac{1}{\epsilon} \int_{\Omega_{\epsilon}} \int_{0}^{T}\left\langle U(t, x)_{-} \mid M U(t, x)_{-}\right\rangle \psi(t) \lambda\left(x^{\prime}\right) d t d x_{n} \mathrm{~d} x^{\prime} .
\end{aligned}
$$

Since $M$ is coercive on $\operatorname{ker}(A+M) \cap \operatorname{Im} A$ (we denote by $\gamma$ the constant of coercivity of $M$ ), equation (9) implies

$$
\alpha \geq \frac{\gamma\left|k_{0}\right|^{2}}{2\left|k_{0}\right|^{2} C_{1} \int_{-\epsilon}^{0} \int_{\mathbb{R}^{n-1} \cap \operatorname{supp} \lambda} \int_{0}^{T} a_{\epsilon}(x) \mathrm{d} t \mathrm{~d} x_{n}} .
$$

We see that $\alpha$ and $\epsilon$ are related by the following inequality

$$
\alpha \geq \frac{C}{\epsilon}
$$

where $C=C\left(\psi, M, A_{i}, \lambda\right)$. Finally,

$$
\begin{aligned}
\left\langle k_{0} \mid M k_{0}\right\rangle \leq & 2 \frac{C_{1} \epsilon}{C} \int_{-\epsilon}^{0} \int_{\mathbb{R}^{n-1} \cap \operatorname{supp} \lambda} \int_{0}^{T}|U(t, x)|^{2} a_{\epsilon}(x) \mathrm{d} t \mathrm{~d} x_{n} \mathrm{~d} x^{\prime} \\
& +\frac{1}{C} \int_{\Omega_{\epsilon}} \int_{0}^{T}\left\langle U(t, x)_{-} \mid M U(t, x)_{-}\right\rangle \psi(t) \lambda\left(x^{\prime}\right) d t d x_{n} \mathrm{~d} x^{\prime} .
\end{aligned}
$$

Since $U \in L_{t, x}^{2}$, the first term of the right hand side of the inequality goes to 0 as $\epsilon$ goes to 0 . The dominated convergence and the fact that $\left\langle U(t, x)_{-} \mid M U(t, x)_{-}\right\rangle \leq\langle U(t, x) \mid M U(t, x)\rangle \leq C|U(t, x)|^{2}$ show us that the second term also goes to 0 as $\epsilon$ goes to 0 . Observe that $M$ is coercive on $\operatorname{ker}(A+M) \cap \operatorname{Im} A$, so by the definition of $k_{0}$ we get the claim.

Remark 8 In the proof of the previous lemma, we crucially use the fact that we were in the case of constant coefficients to get some information on the boundary conditions when we say that $k_{0} \in$ $\operatorname{ker}\left(A_{\nu}+M\right) \cap \operatorname{Im} A_{\nu}$. For the initial conditions, we always have some information since the boundary at the time $t=0$ is flat and the boundary matrix for $t=0$ is the identity matrix. On the contrary, recovering some information in the case where the matrices $A_{\nu}$ and $M$ depending on $x$ and $t$ seems more tricky and is left for further studies.

Using the previous lemmas, we will show that a regular solution in the sense of definition 1 is a solution of the problem 1 


\section{Theorem 1}

Assume that $\Omega$ is a half-plane (8). Assume that $U$ is a dissipative solution and $U$ is a $H_{t, x}^{1}$. Then $U$ is a solution of 1 in the classical sense, i.e.

1. $\partial_{t} U+\sum_{i=1}^{n} A_{i} \partial_{x_{i}} U=f$ in $L_{t, x}^{2}$,

2. $U(0, x)=U_{0}(x)$ in $L_{x}^{2}$,

3. $\left(A_{\nu}-M\right) U(t, x)=0$ in $L^{2}((0, T) \times \partial \Omega)$.

The proof of this theorem follows from the discussion below. First, observe that lemmas 2 and (3) imply respectively the first and the second point of this lemma. Secondly, we need two lemmas to prove that the third point of the previous lemma is true.

\section{Lemma 5}

Assume $\Omega$ is a half-space (8). Let $U \in W^{1, p}((0, T) \times \Omega)(1<p<\infty), \lambda \in \mathcal{D}((0, T))$ and $\beta \in \mathcal{D}\left(\mathbb{R}^{n-1}\right)$. Then the limit when $\epsilon \rightarrow 0$ of

$$
\frac{1}{\epsilon} \int_{-\epsilon}^{0} \int_{0}^{T} \int_{\mathbb{R}^{n-1}} U\left(t, x^{\prime}, x_{n}\right) \lambda(t) \beta\left(x^{\prime}\right) \mathrm{d} x^{\prime} \mathrm{d} t \mathrm{~d} x,
$$

is

$$
\int_{0}^{T} \int_{\mathbb{R}^{n-1}} U\left(t, x^{\prime}, 0\right) \lambda(t) \beta\left(x^{\prime}\right) \mathrm{d} x^{\prime} \mathrm{d} t
$$

Proof : First, observe that the modulus of the quantity

$$
\frac{1}{\epsilon} \int_{-\epsilon}^{0} \int_{0}^{T} \int_{\mathbb{R}^{n-1}} U\left(t, x^{\prime}, x_{n}\right) \lambda(t) \beta\left(x^{\prime}\right) \mathrm{d} x^{\prime} \mathrm{d} t \mathrm{~d} x_{n}-\int_{0}^{T} \int_{\mathbb{R}^{n-1}} U\left(t, x^{\prime}, 0\right) \lambda(t) \beta\left(x^{\prime}\right) \mathrm{d} x^{\prime} \mathrm{d} t,
$$

is bounded above by

$$
\begin{aligned}
& \frac{1}{\epsilon} \int_{-\epsilon}^{0} \int_{0}^{T} \int_{\mathbb{R}^{n-1}}\left|U\left(t, x^{\prime}, x_{n}\right)-U\left(t, x^{\prime}, 0\right)\right| \lambda(t) \beta\left(x^{\prime}\right) \mathrm{d} x^{\prime} \mathrm{d} t \mathrm{~d} x_{n} \\
\leq & \int_{-\epsilon}^{0} \int_{0}^{T} \int_{\mathbb{R}^{n-1}}\left|\frac{U\left(t, x^{\prime}, x_{n}\right)-U\left(t, x^{\prime}, 0\right)}{x_{n}}\right| \lambda(t) \beta\left(x^{\prime}\right) \mathrm{d} x^{\prime} \mathrm{d} t \mathrm{~d} x_{n} \\
\leq & \|\lambda\|_{\infty}\|\beta\|_{\infty} \int_{0}^{T}\left\|\frac{U\left(t, x^{\prime}, x_{n}\right)-U(t, y, 0)}{x_{n}}\right\|_{L^{2}\left((-\epsilon, 0) \times \mathbb{R}^{n-1}\right)} \mathrm{d} x^{\prime} \mathrm{d} t \mathrm{~d} x_{n},
\end{aligned}
$$

But using Hardy's inequality (proved in [20]), we know that for all $t \in(0, T)$

$$
\left\|\frac{U(t)-U\left(t, x_{n}=0\right)}{x_{n}}\right\|_{L^{p}\left(\mathbb{R}_{-}^{n}\right)} \leq \frac{p}{p-1}\left\|\partial_{x_{n}} U(t)\right\|_{L^{p}\left(\mathbb{R}_{-}^{n}\right)},
$$

and consequently $\left(t, x^{\prime}, x_{n}\right) \mapsto \frac{U\left(t, x^{\prime}, x_{n}\right)-U\left(t, x^{\prime}, 0\right)}{x_{n}}$ belongs to $L_{t, x}^{2}$. The lemma follows from the dominated convergence theorem.

To apply the previous result to $U_{+}$, we need the following lemma

\section{Lemma 6}

Assume $\Omega$ is a half-space (8). Suppose that $A_{\nu} U \in H^{1}((0, T) \times \Omega)$. Then $U_{+} \in H^{1}((0, T) \times \Omega)$.

Proof: Since $\operatorname{ker} A_{\nu} \subset \operatorname{ker} M$, there exists a matrix $B$ such that $M=B A_{\nu}$ and consequently $M U$ also belongs to $H^{1}((0, T) \times \Omega)$. But

$$
\left(A_{\nu}-M\right) U=\left(A_{\nu}-M\right)\left(U^{0}+U_{-}+U_{+}\right)=\left(A_{\nu}-M\right) U_{+}=-2 M U_{+} .
$$

We know that $M$ is non-singular on $\operatorname{ker}\left(A_{\nu}+M\right) \cap \operatorname{Im} A_{\nu}$, so $U_{+}$belongs to $H^{1}$. 
Since $U \in H_{t, x}^{1}, A_{\nu} U$ also belongs to $H_{t, x}^{1}$ and thanks to the previous lemma, $U_{+} \in H_{t, x}^{1}$. Now using lemmas 5 and 4 , we deduce that

$$
\int_{0}^{T} \int_{\mathbb{R}^{n-1}} U\left(t, x^{\prime}, 0\right)_{+} \lambda(t) \beta\left(x^{\prime}\right) \mathrm{d} x^{\prime} \mathrm{d} t=0,
$$

for every $\lambda$ and $\beta$ test-functions. Consequently,

$$
U_{+}=0, \quad \text { a.e on }(0, T) \times\left\{x_{n}=0\right\},
$$

the boundary condition is satisfied in the strong sense. Similar arguments apply to the case of the initial condition thanks to lemma 3 .

\section{Existence and uniqueness result in the half-plane}

This section is devoted to prove the following theorem

\section{Theorem 2}

Suppose that $\Omega=\mathbb{R}_{-}^{n}$, the matrices $A_{i}$ and $M$ are supposed to be constant and $A_{n}$ is non singular. For every $f \in L_{t, x}^{2}$ and $U_{0} \in L_{x}^{2}$, there exists a dissipative solution of (1). If $U_{0} \in H_{x}^{1}$, the dissipative solution is unique.

Remark 9 In the previous theorem, we need the $H^{1}$ regularity for the initial condition to satisfy the hypothesis of the theorem 6 but also to know that the function $g$, defined by (17), is $L^{2}$. But, if one can obtain a comparison principle for $L^{2}$ data, as in the case developed in [5] for the whole space, the uniqueness would be easy to obtain. For now, the $H^{1}$ regularity allows us to avoid technical problem about traces on the boundary.

The existence is proven thanks to the classical theory of Friedrichs' systems for regular data and the uniqueness result makes use of mollification in the tangential directions (i.e in the time direction and in the $x^{\prime}$-direction). In order to use the regularity theory (developed in [15]), we assume that

$$
A_{\nu}=A_{n} \text { is non singular. }
$$

In order to prove the existence result, one can use, for example, the theory developed in [3] and [15] for Friedrichs' systems. To do so, we need a matrix $B \in \mathcal{M}_{p \times n}(\mathbb{R})$ where $p$ is the number of negative eigenvalues of $A_{\nu}$ and examine the following initial boundary value problem

$$
\begin{cases}\partial_{t} U+\sum_{i=1}^{n} A_{i} \partial_{x_{i}} U=f, & \text { on }(0, T) \times \Omega, \\ U(0, x)=U_{0}(x), & \text { on } \Omega, \\ B U(t, x)=0, & \text { on }(0, T) \times \partial \Omega,\end{cases}
$$

where $f \in H^{1}([0, T] \times \Omega)$ and $U_{0} \in H^{1}(\Omega)$. We suppose that $U_{0}$ obeys to the following compatibility condition

$$
\forall x \in \partial \Omega, \quad B U_{0}(x)=0 .
$$

To connect our boundary condition and the boundary condition using a matrix $B$, we need that $\operatorname{ker} B=$ $\operatorname{ker}\left(A_{\nu}-M\right)$.

For example, since $\operatorname{ker}\left(A_{\nu}-M\right) \oplus \operatorname{ker}\left(A_{\nu}+M\right)=\mathbb{R}^{n}$, one can take the matrix $B$ that gives the coordinates in a basis of $\operatorname{ker}\left(A_{\nu}+M\right)$ of the projection into the space $\operatorname{ker}\left(A_{\nu}+M\right)$. Since here we are dealing with constant matrices $\left(A_{\nu}\right.$ and $\left.M\right), B$ is also constant.

Under all these assumptions, we can say that there exists a unique solution to the problem (11) and this solution belongs to the space $\mathcal{C}^{1}\left([0, T], L_{x}^{2}\right) \cap \mathcal{C}^{0}\left([0, T], H_{x}^{1}\right)$. Consequently, the initial condition is satisfied in a strong sense and the partial differential equation in (11) has to be understood as an equality in $\mathcal{C}^{0}\left([0, T], L_{x}^{2}\right)$. The boundary condition is satisfied in $\mathcal{C}^{0}\left([0, T], L^{2}(\partial \Omega)^{m}\right)$.

This result can be found in [3] (theorem 9.16). The normality condition and the uniform KreissLopatinskii condition (cf [3]) are automatically fulfilled since $B$ is a strictly dissipative and we are dealing with a symmetric system (cf proposition 4.4 in [3]).

The following theorem tells us that the regular solution of the problem (11) is in fact a regular dissipative solution of (1). 


\section{Theorem 3}

Assuming that $f \in H^{1}([0, T] \times \Omega), U_{0} \in H^{1}(\Omega)$ and condition 12 , there exists a solution to problem (1) in the sense of definition 1

Proof: As we said before, we have

$$
\begin{aligned}
\partial_{t} U+\sum_{i=1}^{n} A_{i} \partial_{x_{i}} U & =f, & & \text { in } \mathcal{C}^{0}\left((0, T), L_{x}^{2}\right), \\
B U(t, x) & =0, & & \text { in } \mathcal{C}^{0}\left((0, T), L_{x}^{2}\right) .
\end{aligned}
$$

If $k$ is a vector of $\mathbb{R}^{n}$ independent of $t$ and $x$, then we also have the following equality

$$
\partial_{t}(U-k)+\sum_{i=1}^{n} A_{i} \partial_{x_{i}}(U-k)=f, \quad \text { in } \mathcal{C}^{0}\left((0, T), L_{x}^{2}\right) .
$$

Consequently, if $\varphi \in W_{c,+}^{1, \infty}((0, T) \times \Omega)$,

$$
\begin{aligned}
& \int_{0}^{T} \int_{\Omega}\left\langle\partial_{t}(U-k) \mid U-k\right\rangle(t, x) \varphi(t, x) \mathrm{d} x \mathrm{~d} t+\int_{0}^{T} \int_{\Omega} \sum_{i=1}^{n}\left\langle U-k \mid A_{i} \partial_{x_{i}}(U-k)\right\rangle(t, x) \varphi(t, x) \mathrm{d} x \mathrm{~d} t \\
= & \int_{0}^{T} \int_{\Omega}\langle f \mid U-k\rangle(t, x) \varphi(t, x) \mathrm{d} x \mathrm{~d} t .
\end{aligned}
$$

Since for almost every $x \in \Omega$, the function $t \mapsto\left\langle\partial_{t}(U-k) \mid U-k\right\rangle(t, x)$ belongs to $\mathcal{C}^{1}(0, T)$, the Green formula and Fubini's theorem give us

$$
\begin{aligned}
\int_{0}^{T} & \int_{\Omega}\left\langle\partial_{t}(U-k) \mid U-k\right\rangle(t, x) \varphi(t, x) \mathrm{d} x \mathrm{~d} t= \\
& \left.\left.\frac{1}{2}\left(\int_{\Omega}|U(T, x)-k|^{2} \varphi(T, x) \mathrm{d} x-\int_{\Omega}\left|U_{0}(x)-k\right|^{2} \varphi(0, x) \mathrm{d} x-\int_{0}^{T} \int_{\Omega} \mid U-k\right)\right|^{2}(t, x) \partial_{t} \varphi(t, x) \mathrm{d} x \mathrm{~d} t\right) .
\end{aligned}
$$

Since the function $x \mapsto U(t, x)$ belongs to $H^{1}(\Omega)$ for every $t \in[0, T]$, it implies that the function $x \mapsto$ $\left\langle U \mid A_{i} U\right\rangle$ belongs to $W^{1,1}(\Omega, \mathbb{R})$ (with $\partial_{x_{i}} 1 / 2\left\langle U \mid A_{i} U\right\rangle=\left\langle U \mid A_{i} \partial_{x_{i}} U\right\rangle$ and consequently admits a trace on $\partial \Omega\left(\right.$ in $\left.L^{1}(\partial \Omega)\right)$. The Green formula (applied to a $W^{1,1}(\Omega, \mathbb{R})$ function and a $W^{1, \infty}\left(\Omega, \mathbb{R}_{+}\right)$function) tells us that

$$
\begin{aligned}
& \int_{\Omega}\left\langle U-k \mid A_{i} \partial_{x_{i}}(U-k)\right\rangle(t, x) \varphi(t, x) \mathrm{d} x \\
= & -\frac{1}{2} \int_{\Omega}\left\langle U-k \mid A_{i}(U-k)\right\rangle(t, x) \partial_{x_{i}} \varphi(t, x) \mathrm{d} x+\frac{1}{2} \int_{\partial \Omega} \nu_{i} T_{1,1}\left\langle U-k \mid A_{i}(U-k)\right\rangle(t, x) \varphi(t, x) \mathrm{d} \sigma(x) .
\end{aligned}
$$

Here $T_{1,1}$ (resp. $T_{1,2}$ ) is the classical trace operator from $W^{1,1}(\Omega)$ to $L^{1}(\partial \Omega)$ (resp. from $W^{1,2}(\Omega)$ to $\left.L^{2}(\partial \Omega)\right)$. By definition of the trace operator and by the density of $\mathcal{C}^{1}(\bar{\Omega})$ in $W^{1,1}(\Omega)\left(\right.$ resp. $\left.W^{1,2}(\Omega)\right)$, we know that for almost every $x \in \partial \Omega$

$$
T_{1,1}\left\langle U-k \mid A_{i}(U-k)\right\rangle(t, x)=\left\langle T_{1,2} U-k \mid A_{i}\left(T_{1,2} U-k\right)\right\rangle .
$$

Consequently,

$$
\begin{aligned}
& \sum_{i=1}^{n} \int_{0}^{T} \int_{\Omega}\left\langle U-k \mid A_{i} \partial_{x_{i}}(U-k)\right\rangle(t, x) \varphi(t, x) \mathrm{d} x \mathrm{~d} t \\
= & -\frac{1}{2} \sum_{i=1}^{n} \int_{0}^{T} \int_{\Omega}\left\langle U-k \mid A_{i}(U-k)\right\rangle(t, x) \partial_{x_{i}} \varphi(t, x) \mathrm{d} x \mathrm{~d} t \\
& +\frac{1}{2} \int_{0}^{T} \int_{\partial \Omega}\left\langle T_{1,2} U-k \mid A_{\nu}\left(T_{1,2} U-k\right)\right\rangle(t, x) \varphi(t, x) \mathrm{d} \sigma(x) \mathrm{d} t .
\end{aligned}
$$

Since we know that $\operatorname{ker} B=\operatorname{ker}\left(A_{\nu}-M\right)$, we can conclude that $T_{1,2} U(x, t) \in \operatorname{ker}\left(A_{\nu}-M\right)$ almost everywhere on $(0, T) \times \partial \Omega$. Lemma 1 tells us that

$$
\begin{aligned}
& \int_{0}^{T} \int_{\partial \Omega}\left\langle T_{1,2} U-k \mid A_{\nu}\left(T_{1,2} U-k\right)\right\rangle(t, x) \varphi(t, x) \mathrm{d} \sigma(x) \mathrm{d} t \\
= & \int_{0}^{T} \int_{\partial \Omega}\left\langle T_{1,2} U-k_{-} \mid M\left(T_{1,2} U-k_{-}\right)\right\rangle(t, x) \mathrm{d} \sigma(x) \mathrm{d} t-\int_{0}^{T} \int_{\partial \Omega}\left\langle k_{+} \mid M k_{+}\right\rangle(t, x) \mathrm{d} \sigma(x) \mathrm{d} t .
\end{aligned}
$$


Finally we deduce from the facts that

$$
\int_{0}^{T} \int_{\partial \Omega}\left\langle T_{1,2} U-k_{-} \mid M\left(T_{1,2} U-k_{-}\right)\right\rangle(t, x) \mathrm{d} \sigma(x) \mathrm{d} t \geq 0
$$

and

$$
\int_{\Omega}|U(T, x)-k|^{2} \varphi(T, x) \mathrm{d} x \geq 0
$$

that we have a dissipative solution to the problem (1) i.e. $\forall k \in \mathbb{R}^{n}, \forall \varphi \in W_{c,+}^{1, \infty}((0, T) \times \Omega)$ we have the following inequality

$$
\begin{aligned}
& \left.\left.\int_{0}^{T} \int_{\Omega}|U(t, x)-k|^{2} \partial_{t} \varphi(t, x)+\sum_{i=1}^{n}\langle U-k| A_{i}(U-k)\right)\right\rangle(t, x) \partial_{x_{i}} \varphi(t, x) \mathrm{d} x \mathrm{~d} t \\
& +\int_{0}^{T} \int_{\Omega} 2\langle f \mid U-k\rangle(t, x) \varphi(t, x) \mathrm{d} x \mathrm{~d} t+\int_{\Omega}\left|U_{0}(x)-k\right|^{2} \varphi(0, x) \mathrm{d} x \\
& +\int_{0}^{T} \int_{\partial \Omega}\left\langle k_{+} \mid M k_{+}\right\rangle(t, x) \mathrm{d} \sigma(x) \mathrm{d} t \geq 0 .
\end{aligned}
$$

Using this result, we can compare regular solutions.

\section{Theorem 4}

Let $U$ and $\tilde{U}$ be two solutions (in the sens of definition 1) associated with $\left(f, U_{0}\right)$ (resp. $\left(\tilde{f}, \tilde{U}_{0}\right)$ ). We suppose that $U$ and $\tilde{U}$ are $H_{t, x}^{1}$. Then there exists a constant $C>0$ (depending only on $T$ ) such that

$$
\|U-\tilde{U}\|_{L_{t, x}^{2}}^{2} \leq C\left(\|f-\tilde{f}\|_{L_{t, x}^{2}}^{2}+\left\|U_{0}-\tilde{U}_{0}\right\|_{L_{x}^{2}}^{2}\right)
$$

Proof: Using the results of the section 2, we know that $U$ and $\tilde{U}$ are solutions of the problem (1) in the classical sense. Consequently, one has

$$
\begin{aligned}
& \int_{0}^{T} \int_{\Omega}\left\langle\partial_{t}(U-\tilde{U}) \mid U-\tilde{U}\right\rangle(t, x) \varphi(t, x) \mathrm{d} x \mathrm{~d} t+\int_{0}^{T} \int_{\Omega} \sum_{i=1}^{n}\left\langle A_{i} \partial_{x_{i}}(U-\tilde{U}) \mid U-\tilde{U}\right\rangle(t, x) \varphi(t, x) \mathrm{d} x \mathrm{~d} t \\
= & \int_{0}^{T} \int_{\Omega}\langle f-\tilde{f} \mid U-\tilde{U}\rangle(t, x) \varphi(t, x) \mathrm{d} x \mathrm{~d} t
\end{aligned}
$$

Taking the function $\varphi:(t, x) \mapsto(T-t) / T$, we get

$$
\begin{aligned}
& \frac{1}{2 T} \int_{0}^{T} \int_{\Omega}|U-\tilde{U}|^{2}(t, x) \mathrm{d} x \mathrm{~d} t+\frac{1}{2} \int_{0}^{T}\left\langle U-\tilde{U} \mid A_{\nu}(U-\tilde{U})\right\rangle(t, 0) \varphi(t, 0) \mathrm{d} t \\
= & \int_{0}^{T} \int_{\Omega}\langle f-\tilde{f} \mid U-\tilde{U}\rangle(t, x) \varphi(t, x) \mathrm{d} x \mathrm{~d} t+\frac{1}{2} \int_{\Omega}\langle U-\tilde{U} \mid U-\tilde{U}\rangle(0, x) \mathrm{d} x,
\end{aligned}
$$

Using the fact that $A_{\nu}(U-\tilde{U})=M(U-\tilde{U})$ on the boundary $x_{n}=0$, we obtain thanks to the fact that $M$ is non-negative

$$
2 \int_{\Omega_{T}}\langle f-\tilde{f} \mid U-\tilde{U}\rangle(t, x) \varphi(t, x) \mathrm{d} x \mathrm{~d} t+\int_{\Omega}\left|U_{0}-\tilde{U}_{0}\right|^{2}(x) \mathrm{d} x \geq \frac{1}{T} \int_{\Omega_{T}}|U-\tilde{U}|^{2}(t, x) \mathrm{d} x \mathrm{~d} t .
$$

The Cauchy-Schwarz inequality leads us to

$$
\frac{1}{T}\|U-\tilde{U}\|_{L_{t, x}^{2}}^{2} \leq 2\|f-\tilde{f}\|_{L_{t, x}^{2}}\|U-\tilde{U}\|_{L_{t, x}^{2}}+\left\|U_{0}-\tilde{U}_{0}\right\|_{L_{x}^{2}}^{2} .
$$

Finally,

$$
\frac{1}{2 T}\|U-\tilde{U}\|_{L_{t, x}^{2}}^{2} \leq 2 T\|f-\tilde{f}\|_{L_{t, x}^{2}}^{2}+\left\|U_{0}-\tilde{U}_{0}\right\|_{L_{x}^{2}}^{2} .
$$

The assertion of the lemma follows easily.

This contraction principle leads to the existence theorem. 


\section{Theorem 5}

If $f \in L_{t, x}^{2}$ and $U_{0} \in L_{x}^{2}$, then there exists a solution of system 1 in the sense of definition 1

Proof : We regularize the problem by taking $f_{n} \in \mathcal{D}((0, T) \times \Omega)$ and $U_{0, n} \in \mathcal{D}(\Omega)$ (remark that automatically we have $B U_{0, n}=0$ on $\partial \Omega$ ) such that $f_{n}$ (resp. $U_{0, n}$ ) tends to $f$ in $L_{t, x}^{2}$ (resp. to $U_{0}$ in $L_{x}^{2}$ ), we denote by $U_{n}$ the solution given by theorem 3 . Then the previous lemma ensures that $\left(U_{n}\right)_{n \in \mathbb{N}}$ is a Cauchy sequence in $L_{t, x}^{2}$ hence it converges to a function $U \in L_{t, x}^{2}$. Passing to the limit when $n$ goes to infinity in the inequality (4) leads to the existence of a solution.

Now, we are going to show a uniqueness result in the case where $U_{0} \in H_{x}^{1}$ and $f \in L_{t, x}^{2}$. Here, we take a solution $U \in L_{t, x}^{2}$ a solution of (1) associated with the function $f \in L_{t, x}^{2}$ and $U_{0} \in H_{x}^{1}$. We are going to regularize $U \in L_{t, x}^{2}$ in the tangential variables $\left(i . e\right.$. the variable $\left.\left(t, x^{\prime}\right)\right)$, so that we will be able to use the tools of theorem 6 which needs more regular solutions.

Now we use convolution in time (with support in $[-\epsilon,-\epsilon / 2]$ and $0<\epsilon<1$ ) and classical convolution in the $x^{\prime}=\left(x_{1}, \cdots, x_{n-1}\right)$ variables. We denote by $\rho_{\epsilon, l}$ the time convolution kernel, $\rho_{\epsilon, x^{\prime}}$ the $x^{\prime}$-convolution kernel. First we extend $U$ for negative times and we denote by $V$ the function $U \mathbb{1}_{(0, T) \times \Omega}+U_{0} \mathbb{1}_{(-\infty, 0) \times \Omega}$, then we define the function $V_{\epsilon}$ as

$$
\begin{aligned}
V_{\epsilon}\left(t, x^{\prime}, x_{n}\right) & =V \star_{t, x^{\prime}} \check{\rho}_{\epsilon}(t, x) \\
& =V \star_{t} \check{\rho_{\epsilon, l} \star_{x}} \check{\check{\rho}_{\epsilon}}(t, x) \\
& =V \star_{t} \check{\rho_{\epsilon, l} \star_{x}} \rho_{\epsilon}(t, x) \\
& =\int_{\mathbb{R}^{n}} V\left(s, y, x_{n}\right) \rho_{\epsilon, l}(s-t) \rho_{\epsilon}\left(y-x^{\prime}\right) \mathrm{d} s \mathrm{~d} y,
\end{aligned}
$$

where $\check{f}$ is the function defined by $\breve{f}(x)=f(-x)$.

\section{Lemma 7}

The function $V_{\epsilon}$ verifies the following properties

1. $V_{\epsilon}$ goes to $U$ in $L_{t, x}^{2}$ when $\epsilon \rightarrow 0$.

2. $V_{\epsilon}$ verifies (in the sense of distribution)

$$
\partial_{t} V_{\epsilon}+\sum_{i=1}^{n} A_{i} \partial_{x_{i}} V_{\epsilon}=f_{\epsilon}
$$

3. for all $\epsilon, V_{\epsilon} \in H^{1}((0, T) \times \Omega)$.

4. for all $\epsilon, V_{\epsilon}(t=0)=U_{0, \epsilon}=U_{0} \star_{x^{\prime}} \rho_{\epsilon}$ (straightforward with the support of $\rho_{\epsilon, l}$ ).

5. $V_{\epsilon+}=0$ on $(0, T) \times \partial \Omega$.

Proof: Let $\varphi$ be in $W_{c,+}^{1, \infty}((-\infty, T) \times \Omega)$. The key observation is the following one : if $U$ is a dissipative solution of (1) with data $f$ and $U_{0}$ then the function $V$ verifies the following inequality

$$
\begin{aligned}
& \int_{-\infty}^{T} \int_{\Omega}\left(|V|^{2}-2\langle V \mid k\rangle\right)(t, x) \partial_{t} \varphi(t, x) \mathrm{d} x \mathrm{~d} t \\
& +\int_{-\infty}^{T} \int_{\Omega} \sum_{i=1}^{n}\left(\left\langle V \mid A_{i} V\right\rangle-2\left\langle V \mid A_{i} k\right\rangle\right)(t, x) \partial_{x_{i}} \varphi(t, x) \mathrm{d} x \mathrm{~d} t \\
& +\int_{-\infty}^{T} \int_{\Omega} 2\langle g \mid V-k\rangle(t, x) \varphi(t, x) \mathrm{d} x \mathrm{~d} t+\int_{-\infty}^{0} \int_{\partial \Omega}\left\langle U_{0} \mid A_{n}\left(U_{0}-k\right)\right\rangle\left(t, x^{\prime}, 0\right) \varphi\left(t, x^{\prime}, 0\right) \mathrm{d} x^{\prime} \mathrm{d} t \\
& +\int_{0}^{T} \int_{\partial \Omega}\left\langle k_{-} \mid M k_{-}\right\rangle\left(t, x^{\prime}, 0\right) \varphi\left(t, x^{\prime}, 0\right) \mathrm{d} x^{\prime} \mathrm{d} t \\
& \geq 0,
\end{aligned}
$$


where

$$
g=f \mathbb{1}_{(0, T) \times \Omega}+2 \sum_{i=1}^{n} A_{i} \partial_{x_{i}} U_{0} \mathbb{1}_{(-\infty, 0) \times \Omega} .
$$

Why (16) is true? We know that $U$ is a solution and consequently, the following inequality is true by definition:

$$
\begin{aligned}
& \int_{-\infty}^{T} \int_{\Omega}\left(|U|^{2}-2\langle U \mid k\rangle\right)(t, x) \partial_{t} \varphi(t, x) \mathrm{d} t \mathrm{~d} x+\int_{-\infty}^{T} \int_{\Omega} \sum_{i=1}^{n}\left(\left\langle U \mid A_{i} U\right\rangle-2\left\langle U \mid A_{i} k\right\rangle\right)(t, x) \partial_{x_{i}} \varphi(t, x) \mathrm{d} t \mathrm{~d} x \\
+ & \int_{-\infty}^{T} \int_{\Omega} 2\langle f \mid U-k\rangle(t, x) \varphi(t, x) \mathrm{d} x \mathrm{~d} t+\int_{\Omega}\left(\left|U_{0}\right|^{2}-2\left\langle U_{0} \mid k\right\rangle\right)(0, x) \varphi(0, x) \mathrm{d} x \\
+ & \int_{0}^{T} \int_{\partial \Omega}\left\langle k_{-} \mid M k_{-}\right\rangle\left(t, x^{\prime}, 0\right) \varphi\left(t, x^{\prime}, 0\right) \mathrm{d} x^{\prime} \mathrm{d} t \geq 0 .
\end{aligned}
$$

But since $U_{0}$ is regular, independent of $t$, we have

$$
\begin{aligned}
& \int_{-\infty}^{0} \int_{\Omega}\left(\left|U_{0}\right|^{2}-2\left\langle U_{0} \mid k\right\rangle\right)(t, x) \partial_{t} \varphi(t, x)+\sum_{i=1}^{n}\left(\left\langle U_{0} \mid A_{i} U_{0}\right\rangle-2\left\langle U_{0} \mid A_{i} k\right\rangle\right)(t, x) \partial_{x_{i}} \varphi(t, x) \mathrm{d} t \mathrm{~d} x \\
= & \int_{\Omega}\left(\left|U_{0}\right|^{2}-2\left\langle U_{0} \mid k\right\rangle\right)(0, x) \varphi(0, x)+2 \int_{-\infty}^{0} \int_{\Omega} \sum_{i=1}^{n}\left(\left\langle A_{i} \partial_{x_{i}} U_{0} \mid U_{0}-k\right\rangle\right)(t, x) \varphi(t, x) \mathrm{d} x \mathrm{~d} t \\
& +\int_{-\infty}^{0} \int_{\partial \Omega}\left\langle A_{n} U_{0} \mid U_{0}-k\right\rangle\left(t, x^{\prime}, 0\right) \varphi\left(t, x^{\prime}, 0\right) \mathrm{d} x^{\prime} \mathrm{d} t .
\end{aligned}
$$

Adding this two inequalities and using the definition of $V$ and $g$ gives us (16). Then the tangential regularization gives us a function $V_{\epsilon}$ satisfying the following inequality

$$
\begin{aligned}
& \int_{\mathbb{R}^{n+1}}\left(\left|V_{\epsilon}\right|^{2}-2\left\langle V_{\epsilon} \mid k\right\rangle\right)(t, x) \partial_{t} \varphi(t, x) \mathrm{d} t \mathrm{~d} x+\int_{\mathbb{R}^{n+1}} \sum_{i=1}^{n}\left(\left\langle V_{\epsilon} \mid A_{i} V_{\epsilon}\right\rangle-2\left\langle V_{\epsilon} \mid A_{i} k\right\rangle\right)(t, x) \partial_{x_{i}} \varphi(t, x) \mathrm{d} t \mathrm{~d} x \\
& +\int_{\mathbb{R}^{n+1}} 2\left\langle g_{\epsilon} \mid V_{\epsilon}-k\right\rangle(t, x) \varphi(t, x) \mathrm{d} t \mathrm{~d} x+\int_{-\infty}^{0} \int_{\mathbb{R}^{n}}\left\langle A_{n} U_{0, \epsilon} \mid U_{0, \epsilon}-k\right\rangle\left(t, x^{\prime}, 0\right) \varphi_{\epsilon}\left(t, x^{\prime}, 0\right) \mathrm{d} x^{\prime} \mathrm{d} t \\
& +\int_{0}^{T} \int_{\mathbb{R}^{n}}\left\langle k_{-} \mid M k_{-}\right\rangle\left(t, x^{\prime}, 0\right) \varphi_{\epsilon}\left(t, x^{\prime}, 0\right) \mathrm{d} x^{\prime} \mathrm{d} t \\
& \geq \int_{\mathbb{R}^{n+1}}\left(\left|U_{\epsilon}\right|^{2}-|U|^{2} \mathbb{1}_{\Omega_{T} \star_{t, x^{\prime}}} \check{\rho_{\epsilon}}\right)(t, x) \partial_{t} \varphi(t, x) \mathrm{d} t \mathrm{~d} x
\end{aligned}
$$

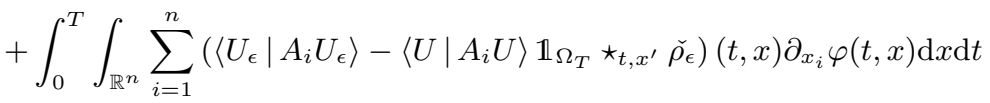

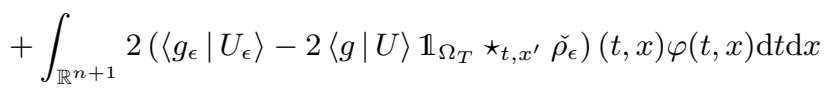

$$
\begin{aligned}
& +\int_{-\infty}^{0} \int_{\mathbb{R}^{n}}\left[\left\langle A_{n} U_{0, \epsilon} \mid U_{0, \epsilon}\right\rangle-\left(\left\langle A_{n} U_{0} \mid U_{0}\right\rangle \star_{x^{\prime}} \check{\rho}_{\epsilon}\right)\left(t, x^{\prime}, 0\right) \varphi_{\epsilon}\right]\left(t, x^{\prime}, 0\right) \mathrm{d} x^{\prime} \mathrm{d} t .
\end{aligned}
$$

The following lemma and its proof ensure that points 2 and 3 are true. As in the proof of lemma 4 , we obtain that $V_{\epsilon+}=0$ on $(0, T) \times \partial \Omega$.

The following lemma explains why we only need to regularize in the $\left(t, x^{\prime}\right)$-directions to get the full $H^{1}$-regularity. 


\section{Lemma 8}

Assume that $A_{n}$ is non singular. Let $U$ be in $L_{t, x}^{2}$ such that $\partial_{t} U, \partial_{1} U, \ldots, \partial_{n-1} U$ belong to $L_{t, x}^{2}$ and we suppose that the following inequality holds for every $k \in \mathbb{R}^{m}$ and $\varphi \in W_{c,+}^{1, \infty}((0, T) \times \Omega)$,

$$
\begin{aligned}
& \int_{0}^{T} \int_{\Omega}|U(t, x)-k|^{2} \partial_{t} \varphi(t, x) \mathrm{d} x \mathrm{~d} t+\int_{0}^{T} \int_{\Omega} \sum_{i=1}^{n}\left\langle U(t, x)-k \mid A_{i}(U(t, x)-k)\right\rangle \partial_{x_{i}} \varphi(t, x) \mathrm{d} x \mathrm{~d} t \\
& +2 \int_{0}^{T} \int_{\Omega}\langle f \mid U-k\rangle \varphi(t, x) \mathrm{d} x \mathrm{~d} t+\int_{\Omega}\left|U_{0}(x)-k\right|^{2} \varphi(0, x) \mathrm{d} x \\
& +\int_{0}^{T} \int_{\partial \Omega}\left\langle k_{+} \mid M(\nu(x)) k_{+}\right\rangle \varphi(t, x) \mathrm{d} x \mathrm{~d} t \\
& \geq \int_{0}^{T} \int_{\Omega} g^{t}(U) \partial_{t} \varphi(t, x)+\sum_{i=1}^{n} g^{i}(U) \partial_{x_{i}} \varphi(t, x) \mathrm{d} x \mathrm{~d} t+\int_{\Omega} g^{0}(U) \varphi(0, x) \mathrm{d} x .
\end{aligned}
$$

where $g^{t}(U), g^{i}(U)$ and $g^{0}(U)$ belong to $L_{t, x}^{2}$ and are independent of $k$. Then $U \in H^{1}((0, T) \times \Omega)$.

Proof: Following the proof of the fact that a solution in the sense of definition 1 is a weak solution, we get that $U$ is a weak solution to the PDE

$$
\partial_{t} U+\sum_{i=1}^{n} A_{i} \partial_{x_{i}} U=f .
$$

Consequently, since $A_{\nu}$ is non-singular by hypothesis, we get that

$$
\partial_{x_{n}} U=A_{n}^{-1}\left(f-\partial_{t} U+\sum_{i=1}^{n-1} A_{i} \partial_{x_{i}} U\right) \in L_{t, x}^{2} .
$$

This implies that $U \in H^{1}((0, T) \times \Omega)$.

Remark 10 The hypothesis that $A_{\nu}$ is invertible is important. In fact in the singular case, there is a loss of regularity for the solution (see [18]).

\section{Theorem 6}

Let $U$ and $\tilde{U}$ be two $L_{t, x}^{2}$ solutions (in the sense of definition 1 ) associated with $\left(f, U_{0}\right) \in L_{t, x}^{2} \times H_{x}^{1}$ and $\left(\tilde{f}, \tilde{U}_{0}\right) \in L_{t, x}^{2} \times H_{x}^{1}$ respectively. Then there exists a constant $C>0$ (depending only on $T$ ) such that

$$
\|U-\tilde{U}\|_{L_{t, x}^{2}}^{2} \leq C\left(\|f-\tilde{f}\|_{L_{t, x}^{2}}^{2}+\left\|U_{0}-\tilde{U}_{0}\right\|_{L_{x}^{2}}^{2}\right)
$$

Remark 11 From the previous theorem, we deduce that there exists a unique solution in the sense of definition 1 of the problem (1) for $f \in L_{t, x}^{2}$ and $U_{0} \in H_{x}^{1}$.

Proof: From $U$ and $\tilde{U}$, we construct their approximations $V_{\epsilon}$ and $\tilde{V}_{\epsilon}$, lemma 7 allows us to mimic the proof of the theorem 6 to get

$$
\left\|V_{\epsilon}-\tilde{V}_{\epsilon}\right\|_{L_{t, x}^{2}}^{2} \leq C\left(\left\|g_{\epsilon}-\tilde{g}_{\epsilon}\right\|_{L_{t, x}^{2}}^{2}+\left\|U_{0, \epsilon}-\tilde{U}_{0 \epsilon}\right\|_{L_{x}^{2}}^{2}\right)
$$

Passing to the limit, since $V$ and $g$ are in $L_{\text {loc }}^{2}((-\infty, T) \times \Omega)^{m}$ then $V_{\epsilon}$ and $g_{\epsilon}$ tends to $V$ and $g$ in $L^{2}((0, T) \times \Omega)^{m}$ (see for example [12]), we get the result.

\section{Comparison with other formulations}

In the literature, there are several ways for taking into account the boundary condition for linear hyperbolic system, for example the initial approach of Friedrichs [9], the formulation developed in [6] by 
Dubois and Le Floch and the maximal and strictly dissipative theories (summarized in the book of Benzoni-Gavage and Serre [3]). We first describe these different approaches which are slightly different from the algebraic point of view, and then compare these formulations on a simple example: the wave equation in 1D. We will see on this example that, except for the maximal dissipative theory, the theory must be modified to take into account the classical Dirichlet boundary condition. This modification can be performed at least formally with the use of a small parameter such that the homogeneous Dirichlet condition is recovered at the limit of a family of dissipative solutions in the sense of definition 1.

\subsection{Friedrichs' approach}

In the seminal work of Friedrichs [9], the boundary condition takes the following form : first he assumes a decomposition of $A_{\nu}=\beta_{-}+\beta_{+}, M$ is now the matrix $\beta_{+}-\beta_{-}$and the boundary condition is $\left(A_{\nu}-M\right) U=0$. Friedrichs also assumes that three conditions (denoted by $\mathrm{III}_{0}, \mathrm{III}_{1}$ and $\mathrm{III}_{2}$ in [9]) are fulfilled by the matrices $\beta_{-}$and $\beta_{+}$

1. $\operatorname{ker}\left(\beta_{-}\right) \oplus \operatorname{ker}\left(\beta_{+}\right)=\mathbb{R}^{m}$.

2. $\operatorname{Im}\left(\beta_{-}\right) \cap \operatorname{Im}\left(\beta_{+}\right)=\{0\}$.

3. ${ }^{t} M+M \geq 0$.

From lemma 1 and the hypothesis that we made on the matrix $M$, we see that all Friedrichs' hypothesis are satisfied in the non-characteristic case (we have already said that the first hypothesis was too restrictive in the characteristic case for example take all the matrices $A_{i}$ equal to zero). As far as assumption 3 is concerned, we have made a restrictive hypothesis : we suppose that $M$ is a symmetric matrix.

\subsection{Dubois-Le Floch's formulation}

Now we describe Dubois-Le Floch's formulation. In order to define admissible boundary condition for nonlinear hyperbolic systems, Dubois and Le Floch (in [6]) also define admissible boundary condition for linear strictly hyperbolic systems. To take account of inhomogeneous boundary condition, they say that the space of admissible condition (for the problem in $(0, T) \times \mathbb{R}_{-}^{n}$ ) is

$$
U_{b}+\operatorname{Vect}\left\{r_{k}, k=p+1, \cdots, m\right\},
$$

where $r_{k}$ is an eigenvector associated with the eigenvalue $\lambda_{k}$ (for $k \geq p+1, \lambda_{k} \geq 0$ and for $k<p+1$, $\lambda_{k}<0$ ) of the boundary matrix $A_{\nu}$. Using our formalism, we can rewrite this space of admissible boundary condition as

$$
U-U_{b} \in \operatorname{ker}\left(A_{\nu}-M\right),
$$

where $M$ is the matrix constructed in the remark 1 with the matrix $P$ associated with the eigenvectors chosen in 18 (recall that these eigenvectors are orthogonal and we may assume without loss of generality that they are orthonormal and consequently that the matrix $P$ is orthogonal). Therefore the type of boundary condition discussed by Dubois and Le Floch is contained in our formalism of boundary condition (in fact strictly contained, see the example of the wave equation).

\subsection{Maximal dissipative and strictly dissipative theories}

We have already used the fact that our formalism can be transformed to use strictly dissipative theory. In these two theories, the boundary condition is taking into account thanks to a matrix $B \in \mathcal{M}_{p \times n}(\mathbb{R})$ where $p$ is the number of negative eigenvalues of $A_{\nu}$. We say that the matrix $B$ is maximal dissipative (in the non-characteristic case) if

$$
U \in \operatorname{ker} B \quad \Rightarrow \quad\left\langle A_{\nu} U \mid U\right\rangle \geq 0,
$$

and $B$ is maximal for that property, i.e. $\operatorname{ker} B$ is not a proper subspace of vector space $V$ such that

$$
U \in V \quad \Rightarrow \quad\left\langle A_{\nu} U \mid U\right\rangle \geq 0,
$$

to get $L^{2}$-well-posedness (theorem 3.2 of 3 ).

This boundary condition (or equivalently the boundary condition used in the work of Rauch, for example in [18) contains more possibilities than the one we use in this paper (in fact, we'll see in the next example that the formalism of [3] allows more boundary conditions than the Friedrichs' approach).

There is also a more stronger condition (see for example [3]): the notion of strictly dissipative boundary condition. A matrix $B$ is strictly dissipative (in the non-characteristic case) if 
1. $U \in \operatorname{ker} B \Rightarrow\left\langle A_{\nu} U \mid U\right\rangle>0$, if $U \neq 0$.

2. $\operatorname{ker} B$ is maximal for the previous property.

3. $B$ is onto.

\subsection{A simple example: the wave equation in 1D}

We consider here the wave equation problem

$$
\partial_{t t} w-\partial_{x x} w=0, \quad \text { on }(0, T) \times \mathbb{R}_{-},
$$

that we rewrite as an hyperbolic problem in dimension $m=2$ as

$$
\partial_{t}\left(\begin{array}{l}
u \\
p
\end{array}\right)-A \partial_{x}\left(\begin{array}{l}
u \\
p
\end{array}\right)=0
$$

where $u=\partial_{t} w, p=\partial_{x} w$ and $A=\left(\begin{array}{ll}0 & 1 \\ 1 & 0\end{array}\right)$. It is classical (see for example [4] section 10.3) that the wave equation is well-posed with the natural following conditions

$$
\begin{aligned}
& w(x, 0)=f(x), \quad \text { on }\{t=0\} \times \mathbb{R}_{-}, \\
& \partial_{t} w(x, 0)=g(x), \quad \text { on }\{t=0\} \times \mathbb{R}_{-} \text {, } \\
& w(0, t)=0, \quad \text { on }(0, T) \times\{x=0\} .
\end{aligned}
$$

Now that the unknowns in 20 are $u=\partial_{t} w$ and $p=\partial_{x} w$, it seems natural to know if one can obtain the boundary condition

$$
u(0, t)=0, \quad \text { on }(0, T) \times\{x=0\}
$$

We are going to show that this kind of boundary condition is not contained in our formalism, Friedrichs' formalism or Dubois-Le Floch's formalism but it can be obtain with the use of a maximal dissipative boundary condition.

First, we are going to show that one can not find two matrices $\beta_{-}$and $\beta_{+}$such that ker $\beta_{-}=$ $\left\{\left(\begin{array}{l}0 \\ p\end{array}\right), p \in \mathbb{R}\right\}$ and the conditions $1,2,3$ are satisfied. Let $\beta_{-}$be the matrix $\left(\begin{array}{ll}a & b \\ c & d\end{array}\right)$.

In fact, the condition $\operatorname{ker} \beta_{-}=\left\{\left(\begin{array}{l}0 \\ p\end{array}\right), p \in \mathbb{R}\right\}$ implies that $b=d=0$. The condition 3 rewrites as

$$
{ }^{t} M+M=\beta_{+}-\beta_{-}+{ }^{t} \beta_{+}-{ }^{t} \beta_{-}=\left(\begin{array}{cc}
-2 a & -2 c-2 \\
-2 c-2 & 0
\end{array}\right) \geq 0
$$

and consequently, it imposes that $c=1$, but then $\beta_{+}=\left(\begin{array}{cc}-a & -1 \\ -2 & 0\end{array}\right)$ is invertible and consequently, the condition 1 can not be fulfilled.

It means that such the boundary condition (21) can not be directly use in the Friedrichs' framework. Since our framework and Dubois-Le Floch's formalism are contained in Friedrichs' framework, one can not expect to use this boundary condition in these theories.

In this particular example, one can see that our framework contains more possibility for the boundary condition than the theory of characteristics of Dubois-Le Floch. Indeed, if we take the example of homogeneous boundary condition, the only possibility from Dubois-Le Floch is that $u+p=0$ on the boundary $x=0$.

Let's determine all the possible matrices $M$ for the wave equation in $1 \mathrm{D}$ that fulfill the conditions of section 2. Necessarily, we have $\operatorname{dim} \operatorname{ker}(A \pm M)=1$ otherwise, either $A-M=0$ or $A+M=0$, these two equalities can not happen since $A$ is neither non-positive nor non-negative. In particular, $\operatorname{det}(A \pm M)=0=a c-(-1 \pm b)^{2}$, subtracting this two equalities tells us that $b=0$. If $b=0$, to insure that $\operatorname{det}(A \pm M)=0$, we get that $\left.a=\frac{1}{c}\right)$. In our formalism, all the matrices $M$ possible are

$$
M_{c}=\left(\begin{array}{cc}
c & 0 \\
0 & \frac{1}{c}
\end{array}\right), \quad \text { with } c>0
$$


The matrix $M_{c}$ is associated with the following boundary condition

$$
u+\frac{p}{c}=0, \quad \text { on }(0, T) \times\{x=0\} .
$$

It allows an infinite number of boundary condition for the wave equation in one dimension, parameterized by $c$.

Finally, we examine the maximal and strictly dissipative boundary conditions in the case of the wave equation. First to use a maximal dissipative boundary condition, one only need to find a matrix $B \in \mathcal{M}_{1 \times 2}(\mathbb{R})$ it simply means that $\operatorname{dim} \operatorname{ker} B=1$. In fact, any matrix $B$ of the form $\left(\begin{array}{ll}a & 0\end{array}\right)$ is maximal dissipative and give the boundary condition (21). One can easily see that the boundary condition $u=0$ can not be strictly dissipative and that in fact the boundary condition (in the general case) that we are considering is such that the space $\operatorname{ker}\left(A_{\nu}-M\right) \cap \operatorname{Im} A_{\nu}$ is strictly dissipative in the sense that the first two previous condition are verified.

Nevertheless, one way to get a formulation that is valid for a $L_{t, x}^{2}$ solution in this particular example would be to send $c$ to $+\infty$ in the dissipative formulation to get a non-dissipative weak formulation. This is very similar to penalization procedure.

One could also modify the definition 1 by restricting the test vectors $k$ to the ones satisfying $k_{+}=0$ if the support of $\varphi$ touches $\partial \Omega$. This formulation seems nevertheless less general than the one studied in this work.

\section{Acknowledgments}

The authors wish to express their thanks to Jean-François Babadjian for many stimulating conversations.

\section{References}

[1] Bardos, C., A. Le Roux, and J.-C. Nédélec (1979). First order quasilinear equations with boundary conditions. Communications in Partial Differential Equations 4(9), 1017-1034.

[2] Bardos, C. and J. Rauch (1982, April). Maximal Positive Boundary Value Problems as Limits of Singular Perturbation Problems. Transactions of the American Mathematical Society 270(2), 377-408.

[3] Benzoni-Gavage, S. and D. Serre (2007). Multi-dimensional Hyperbolic Partial Differential Equations. Oxford University Press.

[4] Brézis, H. (2011). Functional Analysis, Sobolev Spaces and Partial Differential Equations. New-York: Springer.

[5] Després, B., F. Lagoutière, and N. Seguin (2011). Weak solutions to friedrichs systems with convex constraints. Nonlinearity (24), 3055-3081.

[6] Dubois, F. and P. Le Floch (1988). Boundary Conditions for Nonlinear Hyperbolic Systems of Conservation Laws. Journal of Differential Equations 71, 93-122.

[7] Ern, A. and J.-L. Guermond (2006). Discontinuous Galerkin Methods for Friedrichs' systems. I. General Theory. SIAM J. Numeric. Anal. 44(2), 753-778.

[8] Falk, R. S. and G. R. Richter (1999). Explicit Finite Element Methods for Symmetric Hyperbolic Equations. SIAM J. Numeric. Anal. 36(3), 935-952.

[9] Friedrichs, K. (1958). Symmetric Positive Linear Differential Equations. Communications on pure and applied mathematics XI, 333-418.

[10] Kreiss, H.-O. (1970). Initial-Boundary Value Problems for Hyperbolic Equations with Uniformly Characteristic Boundary. Communications on Pure and Applied Mathematics XXIII, 277-298.

[11] Kružkov, S. N. (1970). First order quasilinear equations with several independent variables. Math. URSS-Sb. $10(2), 217-243$.

[12] Leoni, G. (2009). A First Course in Sobolev Spaces. Providence: American mathematical society.

[13] Lions, P.-L. (1996). Mathematical topics in fluid mechanics. Volume 1: Incompressible models. New-York: Oxford University Press. 
[14] Madja, A. and S. Osher (1975). Initial-Boundary Value Problems for Hyperbolic Equations with Uniformly Characteristic Boundary. Communications on Pure and Applied Mathematics XXVIII, 607-675.

[15] Massey, F. and J. Rauch (1974). Differentiability of solutions to hyperbolic initial-boundary value problems. Transactions of the American Mathematical Society 189, 303-318.

[16] Métivier, G. and K. Zumbrun (2005). Hyperbolic boundary value problems for symmetric systems with variable multiplicities. Journal of Differential Equations 211, 61-134.

[17] Otto, F. (1996). Initial-boundary value problem for a scalar conservation law. C. R. Acad. Sci. Paris Sér. I Math. 322(8), 729-734.

[18] Rauch, J. (1985). Symmetric positive systems with boundary characteristic constant multiplicity. Transactions of the American Mathematical Society 291(1), 167-187.

[19] Serre, D. and A. Morando (2005). On the $L^{2}$-well posedness of an initial boundary value problem for the 3D linear elasticity. Communications in Mathematical Sciences 3(4), 575-586.

[20] Tartar, L. (2007). An introduction to Sobolev spaces and interpolation spaces. Berlin: Springer. 